\title{
Stochastic dynamic systems with complex-valued eigensolutions
}

\author{
Sharif Rahman*,†, \\ Department of Mechanical \& Industrial Engineering, The University of Iowa, Iowa City, IA 52242, U.S.A.
}

\begin{abstract}
SUMMARY
A dimensional decomposition method is presented for calculating the probabilistic characteristics of complex-valued eigenvalues and eigenvectors of linear, stochastic, dynamic systems. The method involves a function decomposition allowing lower-dimensional approximations of eigensolutions, Lagrange interpolation of lower-dimensional component functions, and Monte Carlo simulation. Compared with the commonly used perturbation method, neither the assumption of small input variability nor the calculation of the derivatives of eigensolutions is required by the method developed. Results of numerical examples from linear stochastic dynamics indicate that the decomposition method provides excellent estimates of the moments and/or probability densities of eigenvalues and eigenvectors for various cases including large statistical variations of input. Copyright (C) 2007 John Wiley \& Sons, Ltd.
\end{abstract}

Received 20 July 2006; Revised 21 November 2006; Accepted 22 November 2006

KEY WORDS: complex eigenvalue; random eigenvalue; random matrix; decomposition method; univariate decomposition; bivariate decomposition; disc brake system

\section{INTRODUCTION}

Mathematical modelling and simulation to characterize dynamic systems and forecast their evolution in time often lead to large-scale linear or non-linear eigenvalue problems. Generally, the coefficient matrices or operators in these eigenvalue problems are not precisely known because of insufficient information, limited understanding of the underlying phenomena, and inherent randomness. Eigenvalue problems defined for such random matrix, differential, or integral operators are referred to as random eigenvalue problems and are commonly solved by stochastic

\footnotetext{
*Correspondence to: Sharif Rahman, Department of Mechanical \& Industrial Engineering, The University of Iowa, Iowa City, IA 52242, U.S.A.

${ }^{\dagger}$ E-mail: rahman@engineering.uiowa.edu, Website: http://www.engineering.uiowa.edu/ rahman

$\ddagger$ Professor.

Contract/grant sponsor: U.S. National Science Foundation; contract/grant number: DMI-0355487
}

Copyright (C) 2007 John Wiley \& Sons, Ltd. 
methods that determine statistical moments, probability law, and other relevant properties of eigensolutions.

Over the years, many approximate stochastic methods have been developed or examined for solving random eigenvalue problems associated with undamped or proportionally damped stochastic systems. Of these methods, the perturbation methods have dominated the current literature [1-3] because of their ease of implementation and computational efficiency. However, these methods have two major limitations: both the uncertainty of random input and the non-linearity of the random eigenvalue or eigenvector with respect to random input must be small. Methods other than the perturbation methods include the iteration method [1], the Ritz method [4], the crossing theory [5], the stochastic reduced basis [6], the asymptotic method [7], the polynomial chaos expansion [8], and the recently developed dimensional decomposition method [9]. However, most if not all, of these past studies focus strictly on undamped or proportionally damped systems. Although conditions for proportional damping were derived by Caughey and O'Kelly [10], proportional damping is a mathematical construct that allows invoking classical normal modes. If the damping is not proportional, as identified from practical modal testing, linear systems may possess complex modes instead of classical real modes. Complex modes may also appear for undamped or proportionally damped systems with unsymmetric stiffness matrices, for example, when there are frictional springs. However, the consideration of complex modes in stochastic systems has not been widespread. Only recently have non-proportionally damped stochastic systems been examined; in 2004, Adhikari employed the first-order perturbation method to derive the second-moment statistics of complex mode shapes and natural frequencies of simple dynamic systems [11]. However, the perturbation methods require expensive calculation of derivatives of eigensolutions, and more importantly, are limited to small non-linearity of input-output mapping or small input uncertainties. Hence, new stochastic methods for characterizing complex-valued eigensolutions that can manage arbitrarily large non-linearity of input-output mapping and arbitrarily large uncertainties of input are highly desirable.

This paper presents a dimensional decomposition method for predicting the statistical moments and probability density functions of complex-valued eigensolutions of linear stochastic systems. The method can be viewed as an extension of the author's previously developed dimensional decomposition method for analysing stochastic systems with real modes [9]. Section 2 formally defines the random eigenvalue problem involving matrix operators. Section 3 gives a brief exposition of a function decomposition that facilitates lower-dimensional approximations of real and imaginary parts of complex eigensolutions. Section 4 describes the proposed decomposition method and the associated computational effort for calculating the probabilistic characteristics of complex eigenvalues and eigenvectors. In Section 5, three numerical examples from structural dynamics illustrate the method developed. Comparisons have been made with direct Monte Carlo simulation to evaluate the accuracy and computational efficiency of the new method. Finally, Section 6 provides conclusions from this work.

\section{RANDOM EIGENVALUE PROBLEM}

Let $(\Omega, \mathscr{F}, P)$ be a probability space, where $\Omega$ is the sample space, $\mathscr{F}$ is the $\sigma$-algebra of subsets of $\Omega$, and $P$ is the probability measure; $\mathbb{R}^{N}$ and $\mathbb{C}^{N}$ be $N$-dimensional real and complex vector spaces, respectively; and $\mathbb{R}^{N \times N}$ be a set of all $N \times N$, real-valued matrices. Defined on the probability space endowed with the expectation operator $\mathbb{E}$, consider a real-valued $N$-dimensional 
random vector $\mathbf{X}=\left\{X_{1}, \ldots, X_{N}\right\}^{\mathrm{T}} \in \mathbb{R}^{N}$ with the mean vector $\boldsymbol{\mu}_{\mathbf{X}} \equiv \mathbb{E}[\mathbf{X}] \in \mathbb{R}^{N}$, the covariance matrix $\boldsymbol{\Sigma}_{\mathbf{X}} \equiv \mathbb{E}\left[\left(\mathbf{X}-\boldsymbol{\mu}_{\mathbf{X}}\right)\left(\mathbf{X}-\boldsymbol{\mu}_{\mathbf{X}}\right)^{\mathrm{T}}\right] \in \mathbb{R}^{N \times N}$, and the joint probability density function $f_{\mathbf{X}}(\mathbf{x})$ : $\mathbb{R}^{N} \mapsto \mathbb{R}$.

Consider a family of $L \times L$, real-valued, random coefficient matrices $\mathbf{A}_{j}(\mathbf{X}) \in \mathbb{R}^{L \times L} ; j=$ $1, \ldots, J(J>0)$ and a general non-linear function $f$. A non-trivial solution of

$$
f\left(\lambda(\mathbf{X}) ; \mathbf{A}_{1}(\mathbf{X}), \ldots, \mathbf{A}_{J}(\mathbf{X})\right) \phi(\mathbf{X})=\mathbf{0}
$$

if it exists, defines the random eigenvalue $\lambda(\mathbf{X}) \in \mathbb{R}$ or $\mathbb{C}$ and the random eigenvector $\boldsymbol{\phi}(\mathbf{X}) \in \mathbb{R}^{L}$ or $\mathbb{C}^{L}$ of a general non-linear eigenvalue problem. For example, in stochastic dynamics, the equation of motion describing the free vibration of a linear, $L$-degree-of-freedom, discrete system is

$$
\mathbf{M}(\mathbf{X}) \ddot{\mathbf{y}}(t)+\mathbf{C}(\mathbf{X}) \dot{\mathbf{y}}(t)+\mathbf{K}(\mathbf{X}) \mathbf{y}(t)=\mathbf{0}
$$

where the coefficient matrices are the random mass matrix $\mathbf{M}(\mathbf{X}) \in \mathbb{R}^{L \times L}$, the random damping matrix $\mathbf{C}(\mathbf{X}) \in \mathbb{R}^{L \times L}$, and the random stiffness matrix $\mathbf{K}(\mathbf{X}) \in \mathbb{R}^{L \times L} ; \mathbf{y}(t) \in \mathbb{R}^{L}$ is a vector of generalized co-ordinates; and $t \in \mathbb{R}^{+}$is the time, yielding a quadratic eigenvalue problem

$$
\left[\lambda^{2}(\mathbf{X}) \mathbf{M}(\mathbf{X})+\lambda(\mathbf{X}) \mathbf{C}(\mathbf{X})+\mathbf{K}(\mathbf{X})\right] \boldsymbol{\phi}(\mathbf{X})=\mathbf{0}
$$

The probabilistic characteristics of random matrices $\mathbf{M}(\mathbf{X}), \mathbf{C}(\mathbf{X})$, and $\mathbf{K}(\mathbf{X})$ can be derived from the probability law of $\mathbf{X}$. In the present work focused on stochastic dynamics, the following assumptions were made for the properties of coefficient matrices: (1) $\mathbf{M}(\mathbf{X})$ is symmetric and positive semi-definite; (2) $\mathbf{C}(\mathbf{X})$ is not necessarily proportional, i.e. it may in general not be expressed by a linear combination of $\mathbf{M}(\mathbf{X})$ and $\mathbf{K}(\mathbf{X})$; and (3) $\mathbf{K}(\mathbf{X})$ is not necessarily symmetric. The matrix properties allowed by assumptions (2) and/or (3) may generate complex-valued eigensolutions, where complex random eigenvalues $\lambda(\mathbf{X})=\lambda_{R}(\mathbf{X}) \pm \sqrt{-1} \lambda_{I}(\mathbf{X})$ and complex random eigenvectors $\phi(X)=\phi_{R}(\mathbf{X}) \pm \sqrt{-1} \phi_{I}(\mathbf{X})$ occur in conjugate pairs, and subscripts $R$ and $I$ denote their real and imaginary parts, respectively. Both real and imaginary parts of eigensolutions depend on the random input $\mathbf{X}$ via solution of the matrix characteristic equation

$$
\operatorname{det}\left[\lambda^{2}(\mathbf{X}) \mathbf{M}(\mathbf{X})+\lambda(\mathbf{X}) \mathbf{C}(\mathbf{X})+\mathbf{K}(\mathbf{X})\right]=0
$$

and subsequent solution of Equation (3). A major objective in solving a complex-valued random eigenvalue problem is to find the probabilistic characteristics of eigenpairs $\left\{\lambda_{R}^{(i)}(\mathbf{X}) \pm\right.$ $\left.\sqrt{-1} \lambda_{I}^{(i)}(\mathbf{X}), \phi_{R}^{(i)}(\mathbf{X}) \pm \sqrt{-1} \phi_{I}^{(i)}(\mathbf{X})\right\} ; i=1, \ldots, L$ when the probability law of $\mathbf{X}$ is arbitrarily prescribed.

\section{MULTIVARIATE FUNCTION DECOMPOSITION}

Consider the real or imaginary part $\lambda_{m}(\mathbf{x}): \mathbb{R}^{N} \mapsto \mathbb{R}$ of a complex eigenvalue and the real or imaginary part $\boldsymbol{\phi}_{m}(\mathbf{x}): \mathbb{R}^{N} \mapsto \mathbb{R}^{L}$ of a complex eigenvector that depends on $\mathbf{x}=\left\{x_{1}, \ldots, x_{N}\right\}^{\mathrm{T}} \in \mathbb{R}^{N}$, where subscript $m=R$ (real) or $I$ (imaginary). A dimensional decomposition of $\lambda_{m}(\mathbf{x})$ and $\boldsymbol{\phi}_{m}(\mathbf{x})$, 
described by [12-20]

$$
\begin{aligned}
& \lambda_{m}(\mathbf{x})=\underbrace{\lambda_{m, 0}+\sum_{i=1}^{N} \lambda_{m, i}\left(x_{i}\right)}_{=\hat{\lambda}_{m, 1}(\mathbf{x})}+\sum_{\substack{i_{1}, i_{2}=1 \\
i_{1}<i_{2}}}^{N} \lambda_{m, i_{1} i_{2}}\left(x_{i_{1}}, x_{i_{2}}\right)+\cdots+\sum_{\substack{i_{1}, \ldots, i_{S}=1 \\
i_{1}<\cdots<i_{S}}}^{N} \lambda_{m, i_{1} \cdots i_{S}}\left(x_{i_{1}}, \ldots, x_{i_{S}}\right) \\
& =\hat{\lambda}_{m, 2}(\mathbf{x}) \\
& =\hat{\lambda}_{m, S}(\mathbf{x}) \\
& +\cdots+\lambda_{m, 12 \cdots N}\left(x_{1}, \ldots, x_{N}\right)
\end{aligned}
$$

and

$$
\begin{aligned}
& \boldsymbol{\phi}_{m}(\mathbf{x})=\underbrace{\underbrace{\boldsymbol{\phi}_{m, 0}+\sum_{i=1}^{N} \boldsymbol{\phi}_{m, i}\left(x_{i}\right)}_{=\hat{\boldsymbol{\phi}}_{m, 1}(\mathbf{x})}+\sum_{\substack{i_{1}, i_{2}=1 \\
i_{1}<i_{2}}}^{N} \boldsymbol{\phi}_{m, i_{1} i_{2}}\left(x_{i_{1}}, x_{i_{2}}\right)}_{=\hat{\boldsymbol{\phi}}_{m, 2}(\mathbf{x})}+\cdots+\sum_{\substack{i_{1}, \ldots, i_{S}=1 \\
i_{1}<\cdots<i_{S}}}^{N} \boldsymbol{\phi}_{m, i_{1} \cdots i_{S}}\left(x_{i_{1}}, \ldots, x_{i_{S}}\right) \\
&+\cdots+\boldsymbol{\phi}_{m, 12 \cdots N}\left(x_{1}, \ldots, x_{N}\right)
\end{aligned}
$$

can be viewed as a finite hierarchical expansion of an output function in terms of its input variables with increasing dimensions, where $\lambda_{m, 0}$ and $\boldsymbol{\phi}_{m, 0}$ are constants; $\lambda_{m, i}\left(x_{i}\right): \mathbb{R} \mapsto \mathbb{R}$ and $\boldsymbol{\phi}_{m, i}\left(x_{i}\right)$ : $\mathbb{R} \mapsto \mathbb{R}^{L}$ are univariate component functions representing individual contribution to $\lambda_{m}(\mathbf{x})$ and $\phi_{m}(\mathbf{x})$ by input variable $x_{i}$ acting alone; $\lambda_{m, i_{1} i_{2}}\left(x_{i_{1}}, x_{i_{2}}\right): \mathbb{R}^{2} \mapsto \mathbb{R}$ and $\boldsymbol{\phi}_{m, i_{1} i_{2}}\left(x_{i_{1}}, x_{i_{2}}\right): \mathbb{R}^{2} \mapsto \mathbb{R}^{L}$ are bivariate component functions describing the cooperative influence of two input variables $x_{i_{1}}$ and $x_{i_{2}} ; \lambda_{m, i_{1} \cdots i_{S}}\left(x_{i_{1}}, \ldots, x_{i_{S}}\right): \mathbb{R}^{S} \mapsto \mathbb{R}$ and $\boldsymbol{\phi}_{m, i_{1} \cdots i_{S}}\left(x_{i_{1}}, \ldots, x_{i_{S}}\right): \mathbb{R}^{S} \mapsto \mathbb{R}^{L}$ are $S$-variate component functions quantifying the cooperative effects of $S$ input variables $x_{i_{1}}, \ldots, x_{i_{S}}$; and so on. If

$$
\hat{\lambda}_{m, S}(\mathbf{x})=\lambda_{m, 0}+\sum_{i=1}^{N} \lambda_{m, i}\left(x_{i}\right)+\sum_{\substack{i_{1}, i_{2}=1 \\ i_{1}<i_{2}}}^{N} \lambda_{m, i_{1} i_{2}}\left(x_{i_{1}}, x_{i_{2}}\right)+\cdots+\sum_{\substack{i_{1}, \ldots, i_{S}=1 \\ i_{1}<\cdots<i_{S}}}^{N} \lambda_{m, i_{1} \cdots i_{S}}\left(x_{i_{1}}, \ldots, x_{i_{S}}\right)
$$

and

$$
\hat{\boldsymbol{\phi}}_{m, S}(\mathbf{x})=\boldsymbol{\phi}_{m, 0}+\sum_{i=1}^{N} \boldsymbol{\phi}_{m, i}\left(x_{i}\right)+\sum_{\substack{i_{1}, i_{2}=1 \\ i_{1}<i_{2}}}^{N} \boldsymbol{\phi}_{m, i_{1} i_{2}}\left(x_{i_{1}}, x_{i_{2}}\right)+\cdots+\sum_{\substack{i_{1}, \ldots, i_{S}=1 \\ i_{1}<\cdots<i_{S}}}^{N} \boldsymbol{\phi}_{m, i_{1} \cdots i_{S}}\left(x_{i_{1}}, \ldots, x_{i_{S}}\right)
$$

represent general $S$-variate approximations of $\lambda_{m}(\mathbf{x})$ and $\boldsymbol{\phi}_{m}(\mathbf{x})$, respectively, the univariate $(S=1)$ approximations $\hat{\lambda}_{m, 1}(\mathbf{x})$ and $\hat{\boldsymbol{\phi}}_{m, 1}(\mathbf{x})$ and the bivariate $(S=2)$ approximations $\hat{\lambda}_{m, 2}(\mathbf{x})$ and $\hat{\boldsymbol{\phi}}_{m, 2}(\mathbf{x})$ provide two- and three-term approximants, respectively, of the finite decomposition in Equations (7) 
and (8). Similarly, trivariate, quadrivariate, and other higher-variate approximations can be derived by appropriately selecting the value of $S$. The fundamental conjecture underlying this work is that component functions arising in the eigenvalue function decomposition will exhibit insignificant $S$-variate effects cooperatively when $S \rightarrow N$, leading to useful lower-variate approximations of $\lambda_{m}(\mathbf{x})$ and $\boldsymbol{\phi}_{m}(\mathbf{x})$. In the limit, when $S=N, \hat{\lambda}_{m, S}(\mathbf{x})$ and $\hat{\boldsymbol{\phi}}_{m, S}(\mathbf{x})$ converge to the exact functions $\lambda_{m}(\mathbf{x})$ and $\boldsymbol{\phi}_{m}(\mathbf{x})$, respectively. In other words, Equations (7) and (8) generate a hierarchical and convergent sequence of approximations of eigensolutions.

The decomposition presented in Equations (5) or (6) can be traced to the work of Hoeffding [14] in the 1940s, and is well known in the statistics literature under the name analysis of variance (ANOVA) [15]. This decomposition, coined later as the high-dimensional model representation (HDMR) by Rabitz, has been further refined that led to notable contributions in function approximation, including ANOVA-HDMR [16], cut-HDMR [17], and random-sampling (RS)-HDMR [18]. Further generalizations to multi-cut HDMR [19] and monomial preconditioning (mp)-cut-HDMR [20] have also appeared. Recently, the author's group exploited this decomposition technique in calculating statistical moments of response [12] and reliability [13] of uncertain mechanical systems.

\section{DIMENSIONAL DECOMPOSITION METHOD}

\subsection{Lower-dimensional approximations}

For $1 \leqslant S \leqslant N$, consider $S$-variate approximations of $\lambda_{m}(\mathbf{x})$ and $\phi_{m}(\mathbf{x})$, respectively, defined by

$$
\begin{aligned}
\hat{\lambda}_{m, S}(\mathbf{x}) \equiv & \sum_{k=0}^{S}(-1)^{k}\left(\begin{array}{c}
N-S+k-1 \\
k
\end{array}\right) \sum_{\substack{i_{1}, \ldots, i_{S-k}=1 \\
i_{1}<\ldots<i S-k}}^{N} \lambda_{m}\left(c_{1}, \ldots, c_{i_{1}-1}, x_{i_{1}},\right. \\
& \left.c_{i_{1}+1}, \ldots, c_{i_{S-k}-1}, x_{i_{S-k}}, c_{i_{S-k}+1}, \ldots, c_{N}\right)
\end{aligned}
$$

and

$$
\begin{aligned}
\hat{\boldsymbol{\phi}}_{m, S}(\mathbf{x}) \equiv & \sum_{k=0}^{S}(-1)^{k}\left(\begin{array}{c}
N-S+k-1 \\
k
\end{array}\right) \sum_{\substack{i_{1}, \ldots, i_{S-k}=1 \\
i_{1}<\cdots<i}}^{N} \boldsymbol{\phi}_{m}\left(c_{1}, \ldots, c_{i_{1}-1}, x_{i_{1}},\right. \\
& \left.c_{i_{1}+1}, \ldots, c_{i_{S-k}-1}, x_{i_{S-k}}, c_{i_{S-k}+1}, \ldots, c_{N}\right)
\end{aligned}
$$

where $\mathbf{c}=\left\{c_{1}, \ldots, c_{N}\right\}^{\mathrm{T}}$ is a reference point, $\lambda_{m}\left(c_{1}, \ldots, c_{i_{1}-1}, x_{i_{1}}, c_{i_{1}+1}, \ldots, c_{i_{S-k}-1}, x_{i_{S-k}}\right.$, $\left.c_{i_{S-k}+1}, \ldots, c_{N}\right) \quad$ and $\quad \phi_{m}\left(c_{1}, \ldots, c_{i_{1}-1}, x_{i_{1}}, c_{i_{1}+1}, \ldots, c_{i_{S-k}-1}, x_{i_{S-k}}, c_{i_{S-k}+1}, \ldots, c_{N}\right) \quad$ are $(S-k)$ th-dimensional component functions representing $(S-k)$ th-dimensional cooperation among input variables $x_{i_{1}}, \ldots, x_{i_{S-k}}$, where $S=1, \ldots, N$ and $k=0, \ldots, S$. Based on the author's past experience, the mean point of random input defines a suitable reference point. Using a multivariate function theorem developed by Xu and Rahman [12], it can be shown that $\hat{\lambda}_{m, S}(\mathbf{x})$ and $\hat{\boldsymbol{\phi}}_{m, S}(\mathbf{x})$ consist of all terms of the Taylor series of $\lambda_{m}(\mathbf{x})$ and $\phi_{m}(\mathbf{x})$, respectively, that have less than or equal to $S$ variables. The expanded form of Equation (9) or (10), when compared with the Taylor 
expansion of $\lambda_{m}(\mathbf{x})$ or $\boldsymbol{\phi}_{m}(\mathbf{x})$, indicates that the residual error in the $S$-variate approximation includes terms of dimensions $S+1$ and higher. When $S=1$ and 2, Equation (9) or (10) degenerates to univariate or bivariate approximations, respectively. These univariate or bivariate approximations, which include all higher-order univariate or bivariate terms of $\lambda_{m}(\mathbf{x})$ and $\boldsymbol{\phi}_{m}(\mathbf{x})$, should therefore generally provide higher-order representations of eigenvalues than those provided by commonly employed first- or second-order perturbation methods.

The $S$-variate approximation described by Equations (9) or (10), when expanded and further simplified, can be shown to be equivalent to a cut-HDMR. However, if the input is random, the associated component functions are also random. Therefore, further approximation of various component functions in the $S$-variate approximation or cut-HDMR is required for efficient stochastic computation, described as follows.

\subsection{Lagrange interpolation}

Consider $(S-k)$-variate component functions $\lambda_{m}\left(c_{1}, \ldots, c_{i_{1}-1}, x_{i_{1}}, c_{i_{1}+1}, \ldots, c_{i_{S-k}-1}, x_{i_{S-k}}\right.$, $\left.c_{i_{S-k}+1}, \ldots, c_{N}\right)$ and $\phi_{m}\left(c_{1}, \ldots, c_{i_{1}-1}, x_{i_{1}}, c_{i_{1}+1}, \ldots, c_{i_{S-k}-1}, x_{i_{S-k}}, c_{i_{S-k}+1}, \ldots, c_{N}\right)$ in Equations (9) and (10), respectively. If for sample points $x_{i_{1}}=x_{i_{1}}^{\left(j_{1}\right)}, \ldots, x_{i_{S-k}}=x_{i_{S-k}}^{\left(j_{S-k}\right)}, n^{S-k}$ function values $\lambda_{m}\left(c_{1}, \ldots, c_{i_{1}-1}, x_{i_{1}}^{\left(j_{1}\right)}, c_{i_{1}+1}, \ldots, c_{i_{S-k}-1}, x_{i_{S-k}}^{\left(j_{S-k}\right)}, c_{i_{S-k}+1}, \ldots, c_{N}\right) ; j_{1}, j_{S-k}=1, \ldots, n$ and $n^{S-k}$ function values $\boldsymbol{\phi}_{m}\left(c_{1}, \ldots, c_{i_{1}-1}, x_{i_{1}}^{\left(j_{1}\right)}, c_{i_{1}+1}, \ldots, c_{i_{S-k}-1}, x_{i_{S-k}}^{\left(j_{S-k}\right)}, c_{i_{S-k}+1}, \ldots, c_{N}\right) ; j_{1}, j_{S-k}=$ $1, \ldots, n$ are numerically evaluated, the function values for an arbitrary point $\left(x_{i_{1}}, \ldots, x_{i_{S-k}}\right)$ can be interpolated by

$$
\begin{aligned}
\lambda_{m} & \left(c_{1}, \ldots, c_{i_{1}-1}, x_{i_{1}}, c_{i_{1}+1}, \ldots, c_{i_{S-k}-1}, x_{i_{S-k}}, c_{i_{S-k}+1}, \ldots, c_{N}\right) \\
= & \sum_{j_{S-k}=1}^{n} \cdots \sum_{j_{1}=1}^{n} \psi_{j_{1}}\left(x_{i_{1}}\right) \cdots \psi_{j_{S-k}}\left(x_{i_{S-k}}\right) \lambda_{m}\left(c_{1}, \ldots, c_{i_{1}-1}, x_{i_{1}}^{\left(j_{1}\right)},\right. \\
& \left.c_{i_{1}+1}, \ldots, c_{i_{S-k}-1}, x_{i_{S-k}}^{\left(j_{S-k}\right)}, c_{i_{S-k}+1}, \ldots, c_{N}\right)
\end{aligned}
$$

and

$$
\begin{gathered}
\boldsymbol{\phi}_{m}\left(c_{1}, \ldots, c_{i_{1}-1}, x_{i_{1}}, c_{i_{1}+1}, \ldots, c_{i_{S-k}-1}, x_{i_{S-k}}, c_{i_{S-k}+1}, \ldots, c_{N}\right) \\
=\sum_{j_{S-k}=1}^{n} \cdots \sum_{j_{1}=1}^{n} \psi_{j_{1}}\left(x_{i_{1}}\right) \cdots \psi_{j_{S-k}}\left(x_{i_{S-k}}\right) \phi_{m}\left(c_{1}, \ldots, c_{i_{1}-1}, x_{i_{1}}^{\left(j_{1}\right)},\right. \\
\left.c_{i_{1}+1}, \ldots, c_{i_{S-k}-1}, x_{i_{S-k}}^{\left(j_{S-k}\right)}, c_{i_{S-k}+1}, \ldots, c_{N}\right)
\end{gathered}
$$

where

$$
\psi_{j}\left(x_{i}\right)=\frac{\prod_{l=1, l \neq j}^{n}\left(x_{i}-x_{i}^{(l)}\right)}{\prod_{l=1, l \neq j}^{n}\left(x_{i}^{(j)}-x_{i}^{(l)}\right)}
$$


is the $n$-point Lagrange shape function. The combination of Equations (9)-(13) leads to general $S$-variate approximations of eigensolutions, given by

$$
\begin{aligned}
\hat{\lambda}_{m, S}(\mathbf{X}) \cong & \sum_{k=0}^{S}(-1)^{k}\left(\begin{array}{c}
N-S+k-1 \\
k
\end{array}\right) \sum_{\substack{i_{1}, \ldots, i_{S-k}=1 \\
i_{1}<\cdots<i_{S-k}}}^{N} \sum_{j_{S-k}=1}^{n} \cdots \sum_{j_{1}=1}^{n} \psi_{j_{1}}\left(X_{i_{1}}\right) \cdots \psi_{j_{S-k}}\left(X_{i_{S-k}}\right) \\
& \times \lambda_{m}\left(c_{1}, \ldots, c_{i_{1}-1}, x_{i_{1}}^{\left(j_{1}\right)}, c_{i_{1}+1}, \ldots, c_{i_{S-k}-1}, x_{i_{S-k}}^{\left(j_{S-k}\right)}, c_{i_{S-k}+1}, \ldots, c_{N}\right)
\end{aligned}
$$

and

$$
\begin{aligned}
\hat{\boldsymbol{\phi}}_{m, S}(\mathbf{X}) \cong & \sum_{k=0}^{S}(-1)^{k}\left(\begin{array}{c}
N-S+k-1 \\
k
\end{array}\right) \sum_{\substack{i_{1}, \ldots, i_{S-k}=1 \\
i_{1}<\cdots<i_{S-k}}}^{N} \sum_{j_{S-k}=1}^{n} \cdots \sum_{j_{1}=1}^{n} \psi_{j_{1}}\left(X_{i_{1}}\right) \cdots \psi_{j_{S-k}}\left(X_{i_{S-k}}\right) \\
& \times \phi_{m}\left(c_{1}, \ldots, c_{i_{1}-1}, x_{i_{1}}^{\left(j_{1}\right)}, c_{i_{1}+1}, \ldots, c_{i_{S-k}-1}, x_{i_{S-k}}^{\left(j_{S-k}\right)}, c_{i_{S-k}+1}, \ldots, c_{N}\right)
\end{aligned}
$$

which can be employed to generate a convergent sequence of lower-variate approximations of $\lambda_{m}(\mathbf{X})$ or $\boldsymbol{\phi}_{m}(\mathbf{X})$. For example, when $S=1$, the univariate approximations are

$$
\hat{\lambda}_{m, 1}(\mathbf{X}) \cong \sum_{i=1}^{N} \sum_{j=1}^{n} \psi_{j}\left(X_{i}\right) \lambda_{m}\left(c_{1}, \ldots, c_{i-1}, x_{i}^{(j)}, c_{i+1}, \ldots, c_{N}\right)-(N-1) \lambda_{m}(\mathbf{c})
$$

and

$$
\hat{\boldsymbol{\phi}}_{m, 1}(\mathbf{X}) \cong \sum_{i=1}^{N} \sum_{j=1}^{n} \psi_{j}\left(X_{i}\right) \boldsymbol{\phi}_{m}\left(c_{1}, \ldots, c_{i-1}, x_{i}^{(j)}, c_{i+1}, \ldots, c_{N}\right)-(N-1) \boldsymbol{\phi}_{m}(\mathbf{c})
$$

whereas the selection of $S=2$ yields the bivariate approximations

$$
\begin{aligned}
\hat{\lambda}_{m, 2}(\mathbf{X}) \cong & \sum_{\substack{i_{1}, i_{2}=1 \\
i_{1}<i_{2}}}^{N} \sum_{j_{2}=1}^{n} \sum_{j_{1}=1}^{n} \psi_{j_{1}}\left(X_{i_{1}}\right) \psi_{j_{2}}\left(X_{i_{2}}\right) \\
& \times \lambda_{m}\left(c_{1}, \ldots, c_{i_{1}-1}, x_{i_{1}}^{\left(j_{1}\right)}, c_{i_{1}+1}, \ldots, c_{i_{2}-1}, x_{i_{2}}^{\left(j_{2}\right)}, c_{i_{2}+1}, \ldots, c_{N}\right) \\
& -(N-2) \sum_{i=1}^{N} \sum_{j=1}^{n} \psi_{j}\left(X_{i}\right) \lambda_{m}\left(c_{1}, \ldots, c_{i-1}, x_{i}^{(j)}, c_{i+1}, \ldots, c_{N}\right) \\
& +\frac{(N-1)(N-2)}{2} \lambda_{m}(\mathbf{c})
\end{aligned}
$$

and

$$
\hat{\boldsymbol{\phi}}_{m, 2}(\mathbf{X}) \cong \sum_{\substack{i_{1}, i_{2}=1 \\ i_{1}<i_{2}}}^{N} \sum_{j_{2}=1}^{n} \sum_{j_{1}=1}^{n} \psi_{j_{1}}\left(X_{i_{1}}\right) \psi_{j_{2}}\left(X_{i_{2}}\right)
$$




$$
\begin{aligned}
& \times \phi_{m}\left(c_{1}, \ldots, c_{i_{1}-1}, x_{i_{1}}^{\left(j_{1}\right)}, c_{i_{1}+1}, \ldots, c_{i_{2}-1}, x_{i_{2}}^{\left(j_{2}\right)}, c_{i_{2}+1}, \ldots, c_{N}\right) \\
& -(N-2) \sum_{i=1}^{N} \sum_{j=1}^{n} \psi_{j}\left(X_{i}\right) \phi_{m}\left(c_{1}, \ldots, c_{i-1}, x_{i}^{(j)}, c_{i+1}, \ldots, c_{N}\right) \\
& +\frac{(N-1)(N-2)}{2} \boldsymbol{\phi}_{m}(\mathbf{c})
\end{aligned}
$$

Trivariate and other higher-variate approximations can be generated in a similar manner, but due to their higher cost, only univariate and bivariate approximations are considered in this paper.

The sample points required by Lagrange interpolations can be selected based on the mean and standard deviation of the random input. For example, if $n$ sample points are uniformly deployed in the direction of $X_{i}$, which has mean $\mu_{i}$ and standard deviation $\sigma_{i}$, the sample points for the univariate approximation are: $\left(\ldots, \mu_{i}-2 \sigma_{i}, \mu_{i}-\sigma_{i}, \mu_{i}, \mu_{i}+\sigma_{i}, \mu_{i}+2 \sigma_{i}, \ldots\right)$. Other selections entailing a sparser uniform spacing or a non-uniform spacing can be envisioned. Nonetheless, the number and location of sample points should be selected in such a way that the resulting approximation is insensitive to further refinement. From the author's past experience, a uniform spacing with five sample points works well unless the input uncertainty or the non-linearity of the component function is overly large, in which case a larger number of sample points may be required. The appropriateness of selected sample points will be numerically evaluated in a forthcoming section.

\subsection{Monte Carlo simulation}

Once the Lagrange shape functions $\psi_{j_{1}}\left(x_{i_{1}}\right), \ldots, \psi_{j_{S-k}}\left(x_{i_{S-k}}\right)$, eigenvalue coefficients $\lambda_{m}\left(c_{1}, \ldots\right.$, $\left.c_{i_{1}-1}, x_{i_{1}}^{\left(j_{1}\right)}, c_{i_{1}+1}, \ldots, c_{i_{S-k}-1}, x_{i_{S-k}}^{\left(j_{S-k}\right)}, c_{i_{S-k}+1}, \ldots, c_{N}\right)$, and eigenvector coefficients $\boldsymbol{\phi}_{m}\left(c_{1}, \ldots\right.$, $\left.c_{i_{1}-1}, x_{i_{1}}^{\left(j_{1}\right)}, c_{i_{1}+1}, \ldots, c_{i_{S-k}-1}, x_{i_{S-k}}^{\left(j_{S-k}\right)}, c_{i_{S-k}+1}, \ldots, c_{N}\right) ; S=1, \ldots, N ; k=0, \ldots, S ; j_{1}, j_{S-k}=1$, $\ldots, n$, are generated, Equations (14)-(19) provide explicit approximations of random eigensolutions $\left\{\lambda_{R}^{(i)}(\mathbf{X}) \pm \sqrt{-1} \lambda_{I}^{(i)}(\mathbf{X}), \phi_{R}^{(i)}(\mathbf{X}) \pm \sqrt{-1} \phi_{I}^{(i)}(\mathbf{X})\right\} ; i=1, \ldots, L$, in terms of random input $\mathbf{X}$. Therefore, any probabilistic characteristic of eigensolutions, including their moments and probability density functions, can be easily evaluated by performing Monte Carlo simulation on Equations (14)-(19). Since Equations (14)-(19) do not require solving additional matrix equations, the embedded Monte Carlo simulation can be efficiently conducted for any sample size. Furthermore, statistical moments obtained from Equations (14)-(19) can be employed in reconstructing probability density function using the maximum entropy approach [21].

The proposed methods involving univariate (Equations (16) and (17)) or bivariate (Equations (18) and (19)) approximations, $n$-point Lagrange interpolation, and associated Monte Carlo simulation are defined as the univariate or bivariate decomposition methods in this paper. No expensive calculation of partial derivatives of eigensolutions is required.

\subsection{Computational effort}

In reference to Equations (16) and (18), the univariate and bivariate decomposition methods require evaluations of deterministic coefficients $\lambda_{m}(\mathbf{c}), \lambda_{m}\left(c_{1}, \ldots, c_{i-1}, x_{i}^{(j)}, c_{i+1}, \ldots, c_{N}\right)$, and $\lambda_{m}\left(c_{1}, \ldots, c_{i_{1}-1}, x_{i_{1}}^{\left(j_{1}\right)}, c_{i_{1}+1}, \ldots, c_{i_{2}-1}, x_{i_{2}}^{\left(j_{2}\right)}, c_{i_{2}+1}, \ldots, c_{N}\right)$ for $m=R$ or $I ; i, i_{1}, i_{2}=1, \ldots, N$; 
and $j, j_{1}, j_{2}=1, \ldots, n$. Hence, the computational effort to obtain these coefficients can be viewed as numerically solving the associated deterministic characteristic equations

$$
\begin{gathered}
\operatorname{det}\left[\lambda^{2}(\mathbf{c}) \mathbf{M}(\mathbf{c})+\lambda(\mathbf{c}) \mathbf{C}(\mathbf{c})+\mathbf{K}(\mathbf{c})\right]=0 \\
\operatorname{det}\left[\lambda^{2}\left(c_{1}, \ldots, c_{i-1}, x_{i}^{(j)}, c_{i+1}, \ldots, c_{N}\right) \mathbf{M}\left(c_{1}, \ldots, c_{i-1}, x_{i}^{(j)}, c_{i+1}, \ldots, c_{N}\right)\right. \\
+\lambda\left(c_{1}, \ldots, c_{i-1}, x_{i}^{(j)}, c_{i+1}, \ldots, c_{N}\right) \mathbf{C}\left(c_{1}, \ldots, c_{i-1}, x_{i}^{(j)}, c_{i+1}, \ldots, c_{N}\right) \\
\left.+\mathbf{K}\left(c_{1}, \ldots, c_{i-1}, x_{i}^{(j)}, c_{i+1}, \ldots, c_{N}\right)\right]=0, \quad i=1, \ldots, N, \quad j=1, \ldots, n
\end{gathered}
$$

and

$$
\begin{aligned}
\operatorname{det} & \lambda^{2}\left(c_{1}, \ldots, c_{i_{1}-1}, x_{i_{1}}^{\left(j_{1}\right)}, c_{i_{1}+1}, \ldots, c_{i_{2}-1}, x_{i_{2}}^{\left(j_{2}\right)}, c_{i_{2}+1}, \ldots, c_{N}\right) \\
\times & \mathbf{M}\left(c_{1}, \ldots, c_{i_{1}-1}, x_{i_{1}}^{\left(j_{1}\right)}, c_{i_{1}+1}, \ldots, c_{i_{2}-1}, x_{i_{2}}^{\left(j_{2}\right)}, c_{i_{2}+1}, \ldots, c_{N}\right) \\
+ & \lambda\left(c_{1}, \ldots, c_{i_{1}-1}, x_{i_{1}}^{\left(j_{1}\right)}, c_{i_{1}+1}, \ldots, c_{i_{2}-1}, x_{i_{2}}^{\left(j_{2}\right)}, c_{i_{2}+1}, \ldots, c_{N}\right) \\
\times & \mathbf{C}\left(c_{1}, \ldots, c_{i_{1}-1}, x_{i_{1}}^{\left(j_{1}\right)}, c_{i_{1}+1}, \ldots, c_{i_{2}-1}, x_{i_{2}}^{\left(j_{2}\right)}, c_{i_{2}+1}, \ldots, c_{N}\right) \\
+ & \left.\mathbf{K}\left(c_{1}, \ldots, c_{i_{1}-1}, x_{i_{1}}^{\left(j_{1}\right)}, c_{i_{1}+1}, \ldots, c_{i_{2}-1}, x_{i_{2}}^{\left(j_{2}\right)}, c_{i_{2}+1}, \ldots, c_{N}\right)\right]=0 \\
& i_{1}, i_{2}=1, \ldots, N, \quad j_{1}, j_{2}=1, \ldots, n
\end{aligned}
$$

at several deterministic input defined by user-selected sample points. Therefore, the total cost for the univariate method entails a maximum of $n N+1$ solutions of the matrix characteristic equation, and a maximum of $N(N-1) n^{2} / 2+n N+1$ solutions of the matrix characteristic equation are required for the bivariate method. If the sample points include a common point in each co-ordinate $x_{i}$ (see Example section), the numbers of such solutions reduce to $(n-1) N+1$ and $N(N-1)(n-1)^{2} / 2+(n-1) N+1$ for the univariate and bivariate methods, respectively.

Equations (9)-(22) are the result of a generalization of author's previous work on real eigenvalue problems [9]. Equations (9)-(22) will be employed in obtaining stochastic solutions of complexvalued eigenproblems in dynamic systems, including solving a large-scale eigenvalue problem from the automotive industry.

\section{NUMERICAL EXAMPLES}

Three numerical examples involving linear dynamics of a spring-mass-damper system, a cantilever beam, and an automotive disc brake system are presented to illustrate the decomposition method. Comparisons have been made with the direct Monte Carlo simulation to evaluate the accuracy and efficiency of the proposed method. For the first two examples, the eigenvalues were calculated by an IMSL subroutine, which employs the state-space approach [22] and a hybrid double-shifted LRQR algorithm [23]. A subspace projection algorithm embedded in the commercial finite element code ABAQUS (Version 6.5) [24] was employed to obtain eigenvalues for the third example. 


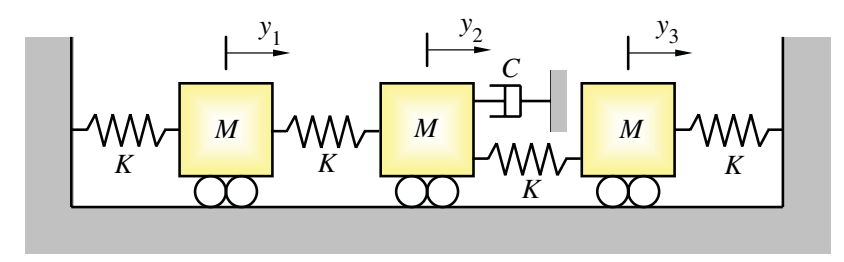

Figure 1. A three-degree-of-freedom spring-mass-damper system.

In all three examples, the univariate and/or bivariate decompositions were formulated in the Gaussian image (u space) of the original space ( $\mathbf{x}$ space) of random input. The reference point $\mathbf{c}$ was fixed at the mean input in the $\mathbf{u}$ space. A 5-point $(n=5)$ Lagrange interpolation involving five sample points $\left(c_{1}, \ldots, c_{i-1}, u_{i}^{(j)}, c_{i+1}, \ldots, c_{N}\right)$ and $\left(c_{1}, \ldots, c_{i_{1}-1}, u_{i_{1}}^{\left(j_{1}\right)}, c_{i_{1}+1}, \ldots, c_{i_{2}-1}, u_{i_{2}}^{\left(j_{2}\right)}\right.$, $\left.c_{i_{2}+1}, \ldots, c_{N}\right)$ in the $\mathbf{u}$ space were chosen with $c_{i}=0$ and uniformly distributed points $u_{i}^{(j)}$ or $u_{i_{1}}^{\left(j_{1}\right)}$ or $u_{i_{2}}^{\left(j_{2}\right)}=-2,-1,0,1,2$. Hence, for both moment and probability density analyses, $(n-1) N+1$ and $(n-1)^{2} N(N-1) / 2+(n-1) N+1$ solutions of the matrix characteristic equation are involved in the univariate and bivariate methods, respectively.

\subsection{Example 1-dynamics of a three-degree-of-freedom spring-mass-damper system}

As shown in Figure 1, consider a three-degree-of-freedom, non-proportionally damped, springmass-damper system with random mass, damping, and stiffness matrices

$$
\mathbf{M}(\mathbf{X})=\left[\begin{array}{ccc}
M(\mathbf{X}) & 0 & 0 \\
0 & M(\mathbf{X}) & 0 \\
0 & 0 & M(\mathbf{X})
\end{array}\right] \in \mathbb{R}^{3 \times 3}, \quad \mathbf{C}(\mathbf{X})=\left[\begin{array}{ccc}
0 & 0 & 0 \\
0 & C(\mathbf{X}) & 0 \\
0 & 0 & 0
\end{array}\right] \in \mathbb{R}^{3 \times 3}
$$

and

$$
\mathbf{K}(\mathbf{X})=\left[\begin{array}{ccc}
2 K(\mathbf{X}) & -K(\mathbf{X}) & 0 \\
-K(\mathbf{X}) & 2 K(\mathbf{X}) & -K(\mathbf{X}) \\
0 & -K(\mathbf{X}) & 2 K(\mathbf{X})
\end{array}\right] \in \mathbb{R}^{3 \times 3}
$$

respectively, where $M(\mathbf{X})=\mu_{M} X_{1}$ is the random mass, $C(\mathbf{X})=\mu_{C} X_{2}$ is the random linear viscous damping coefficient, $K(\mathbf{X})=\mu_{K} X_{3}$ is the random linear spring stiffness, $\mu_{M}=1 \mathrm{~kg}$, $\mu_{C}=0.3 \mathrm{~N} \mathrm{~s} / \mathrm{m}$, and $\mu_{K}=1 \mathrm{~N} / \mathrm{m}$. The random input $\mathbf{X}=\left\{X_{1}, X_{2}, X_{3}\right\}^{\mathrm{T}} \in \mathbb{R}^{3}$ is an independent, trivariate, lognormal random vector with mean $\boldsymbol{\mu}_{\mathbf{X}} \equiv \mathbb{E}[\mathbf{X}]=\mathbf{1} \in \mathbb{R}^{3}$, covariance matrix $\boldsymbol{\Sigma}_{\mathbf{X}} \equiv \mathbb{E}[(\mathbf{X}-$ $\left.\left.\boldsymbol{\mu}_{\mathbf{X}}\right)\left(\mathbf{X}-\boldsymbol{\mu}_{\mathbf{X}}\right)^{\mathrm{T}}\right]=v^{2} \mathbf{I} \in \mathbb{R}^{3 \times 3}$, and coefficient of variation $v=0.25$ or 0.5 . Two cases involving moderate $(v=0.25)$ and large $(v=0.5)$ input uncertainties were studied. The sample size for the direct Monte Carlo simulation and the decomposition method was 10000 . The purpose of this example is to predict second-moment properties of eigenvalues by the proposed decomposition method and compare results with those obtained from the direct Monte Carlo simulation.

Due to non-proportional damping, the spring-mass-damper system possesses complex modes with six complex eigenvalues denoted by $\left\{\lambda_{R}^{(i)}(\mathbf{X}) \pm \sqrt{-1} \lambda_{I}^{(i)}(\mathbf{X})\right\} ; i=1, \ldots, 3$. Let 
$\mathbf{Y}=\left\{\lambda_{R}^{(1)}, \lambda_{R}^{(2)}, \lambda_{R}^{(3)}, \lambda_{I}^{(1)}, \lambda_{I}^{(2)}, \lambda_{I}^{(3)}\right\}^{\mathrm{T}} \in \mathbb{R}^{6}$ define a response vector comprising all three real and all three imaginary parts of eigenvalues. Table I presents the mean vector $\left(\boldsymbol{\mu}_{\mathbf{Y}}\right)$ and the covariance matrix $\left(\boldsymbol{\Sigma}_{\mathbf{Y}}\right)$ of $\mathbf{Y}$ for both magnitudes of input uncertainties, calculated by the univariate and bivariate decomposition methods entailing only 13 and 61 solutions of the matrix characteristic equation, respectively. A direct Monte Carlo simulation by repeatedly solving the matrix characteristic equation was also performed to generate corresponding benchmark results, which are listed in Table I. The second rows of $\boldsymbol{\mu}_{\mathbf{Y}}$ and $\boldsymbol{\Sigma}_{\mathbf{Y}}$ contain zero elements, because the third and fourth eigenvalues associated with the second mode are purely imaginary (i.e. $\lambda_{R}^{(2)}(\mathbf{X})=0$ ). From the results of Table I, the univariate and bivariate decomposition methods provide almost the same benchmark estimates of means for both magnitudes of input uncertainties. When comparing covariance properties for moderate input uncertainties $(v=0.25)$, the maximum errors in approximate variances measured with respect to the direct Monte Carlo estimates are about $10 \%$ by the univariate method and $0 \%$ by the bivariate method. The $0 \%$ error by the bivariate method is due to five significant digits retained in comparing numerical values. Nonetheless, both versions of the decomposition method provide satisfactory estimates of the second-moment properties of eigenvalues for moderate input uncertainties in this particular example. However, when there are large input uncertainties $(v=0.5)$, the maximum error by the univariate method rises to $37 \%$ and is no longer acceptable. In contrast, the bivariate method yields significantly improved results with errors less than $5 \%$. The error can be further reduced by invoking a higher-variate decomposition method if desired. This improvement is possible due to the hierarchical sequence of approximations in the dimensional decomposition method. Nevertheless, bivariate or higher-variate decomposition methods may be required to account for large uncertainties of input. It is worth noting that the commonly employed perturbation methods are not expected to provide acceptable results for both magnitudes of input uncertainties considered in this example [1-3]. An input coefficient of variation, as low as $10 \%$, was required to yield satisfactory second-moment estimates of complex-valued eigensolutions by perturbation methods [11].

The statistics of $\mathbf{Y}$ predicted by the decomposition method were recalculated for a different and new selection of sample points: $u_{i}^{(j)}$ or $u_{i_{1}}^{\left(j_{1}\right)}$ or $u_{i_{2}}^{\left(j_{2}\right)}=-3,-0.5,0,0.5,3{ }^{\S}$ The statistics obtained using the new sample points are almost the same as listed in Table I. For example, the maximum difference in the variances calculated using new and old sample points by univariate or bivariate methods is only $0.3 \%$. Such a comparison of results, although presented here for a specific problem, shows that the final result is not so sensitive to the selection of sample points.

\subsection{Example 2-flexural vibration of a free-standing beam}

For the next example, the vibration of a tall, free-standing beam [22] shown in Figure 2(a) was studied. Figure 2(b) represents a lumped-parameter model of the beam, which comprises seven rigid, massless links hinged together. The mass of the beam is represented by seven random point masses $M$ located at the centre of each link. No damping was assumed except at the bottom joint, where the random, rotational, viscous damping coefficient due to the foundation pad is $C$. The random rotational stiffness at the bottom of the beam, controlled by the lower half of the bottom link and the flexibility of the foundation pad, is $K$. The independent random variables $M, C$, and $K$ are lognormally distributed with respective means $3000 \mathrm{~kg}, 2 \times 10^{7} \mathrm{~N} \mathrm{~m} \mathrm{~s} / \mathrm{rad}$, and

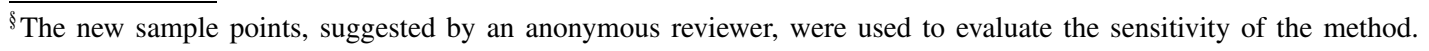




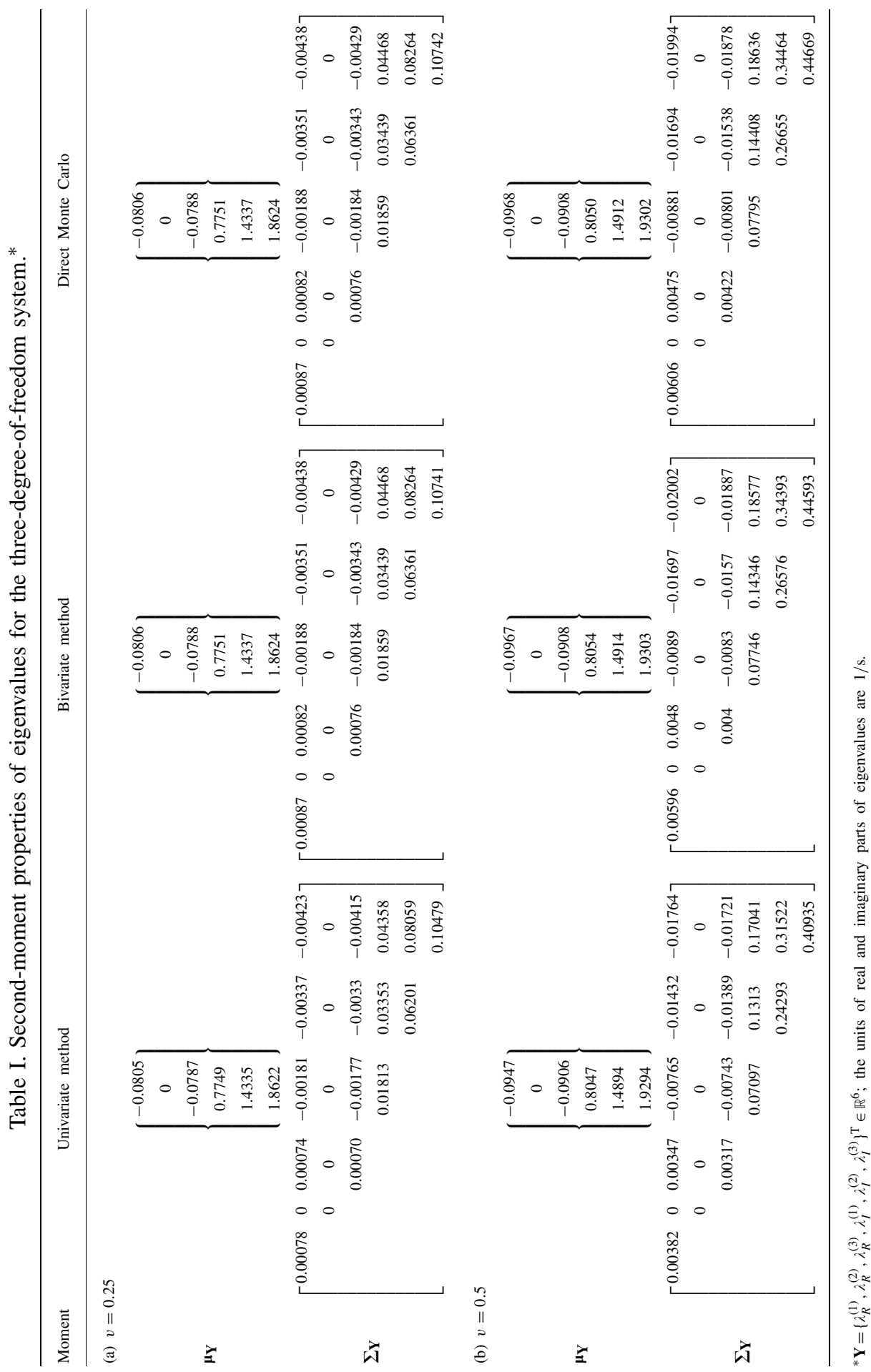

Copyright () 2007 John Wiley \& Sons, Ltd. 


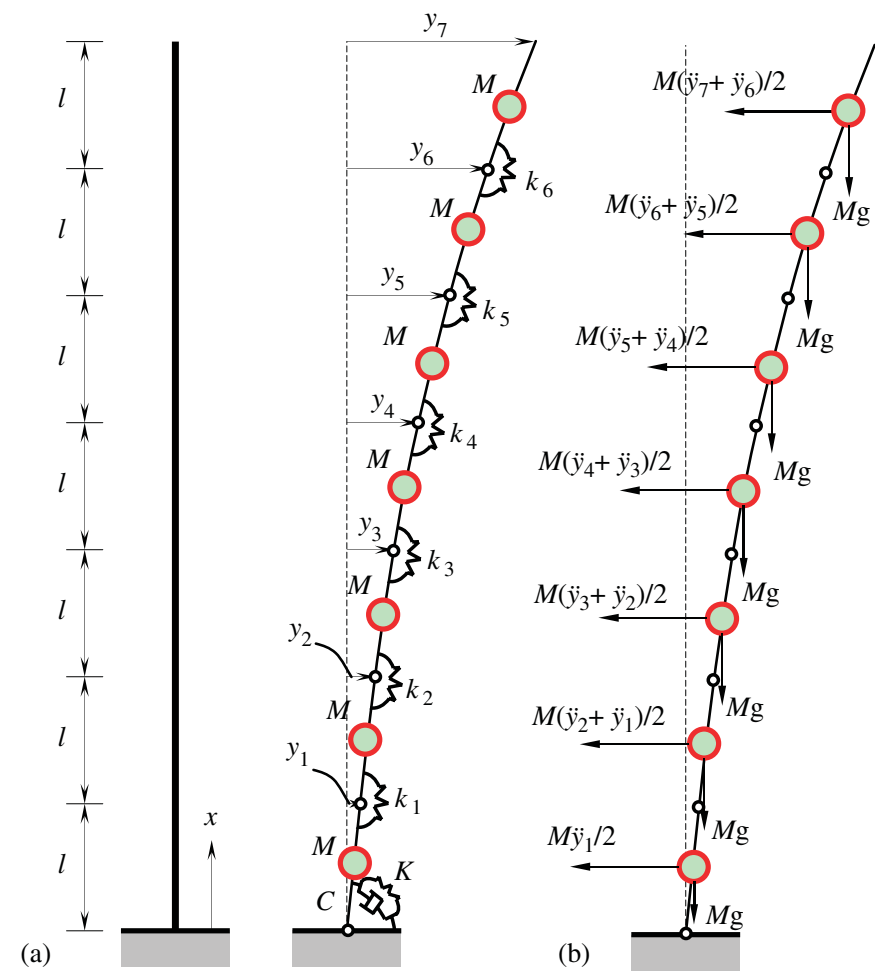

Figure 2. Vibration of a free-standing beam: (a) continuous representation; and (b) seven-degree-of-freedom discrete model.

$2 \times 10^{9} \mathrm{Nm} / \mathrm{rad}$ and have a $20 \%$ coefficient of variation. The flexural rigidity of the beam is represented by six rotational springs between links with stiffnesses $k_{i} \equiv k\left(x_{i}\right) ; i=1, \ldots, 6$, where $x_{i}=i l ; i=1, \ldots, 6$, and $l=6 \mathrm{~m}$. The spatially varying spring stiffness $k(x)=c_{\alpha} \exp [\alpha(x)]$ is an independent, homogeneous, lognormal random field with mean $\mu_{k}=2 \times 10^{9} \mathrm{~N} \mathrm{~m} / \mathrm{rad}$; variance $\sigma_{k}^{2}=2 \times 10^{9} v_{k}^{2} \mathrm{~N}^{2} \mathrm{~m}^{2} / \mathrm{rad}^{2}$; and coefficient of variation $v_{k}=0.2$; where $c_{\alpha}=\mu_{k} / \sqrt{1+v_{k}^{2}}$ and $\alpha(x)$ is a zero-mean, homogeneous, Gaussian random field with variance $\sigma_{\alpha}^{2}=\ln \left(1+v_{k}^{2}\right)$ and covariance function $\Gamma_{\alpha}(u) \equiv \mathbb{E}[\alpha(x) \alpha(x+u)]=\sigma_{\alpha}^{2} \exp (-|u| / l)$. A discretization of $\alpha(x)$ yields the zero-mean Gaussian random vector $\alpha \equiv\left\{\alpha_{1}, \ldots, \alpha_{6}\right\}^{\mathrm{T}} \equiv\{\alpha(l), \ldots, \alpha(6 l)\}^{\mathrm{T}} \in \mathbb{R}^{6}$ with the covariance matrix $\boldsymbol{\Sigma}_{\alpha}=\left[\mathbb{E}\left(\alpha_{p} \alpha_{q}\right)\right] ; p, q=1, \ldots, 6$, where $\mathbb{E}\left(\alpha_{p} \alpha_{q}\right)=\mathbb{E}(\alpha(p l) \alpha(q l))=\Gamma_{\alpha}((q-p) l)$, leading to complete statistical characterization of spring stiffnesses $k_{i}=c_{\alpha} \exp \left[\alpha_{i}\right]$. Therefore, the input random vector $\mathbf{X}=\left\{M, C, K, \alpha_{1}, \ldots, \alpha_{6}\right\}^{\mathrm{T}} \in \mathbb{R}^{9}$ includes nine random variables in this example.

Rotational equilibrium of various links about their appropriate hinges, depicted in Figure 2(b), leads to the standard linear equation of motion (Equation (2)), where $\mathbf{y}=\left\{y_{1}, \ldots, y_{7}\right\}^{\mathrm{T}} \in \mathbb{R}^{7}$ is the vector of displacements. The $7 \times 7$ random mass, random damping, and random stiffness matrices are defined in Appendix A.

Due to non-proportional damping, the discrete beam model yields 14 complex eigenvalues $\lambda^{(i)}(\mathbf{X})=\lambda_{R}^{(i)}(\mathbf{X}) \pm \sqrt{-1} \lambda_{I}^{(i)}(\mathbf{X}) ; i=1, \ldots, 7$. Figures $3(\mathrm{a})$ and (b) plot predicted means and 

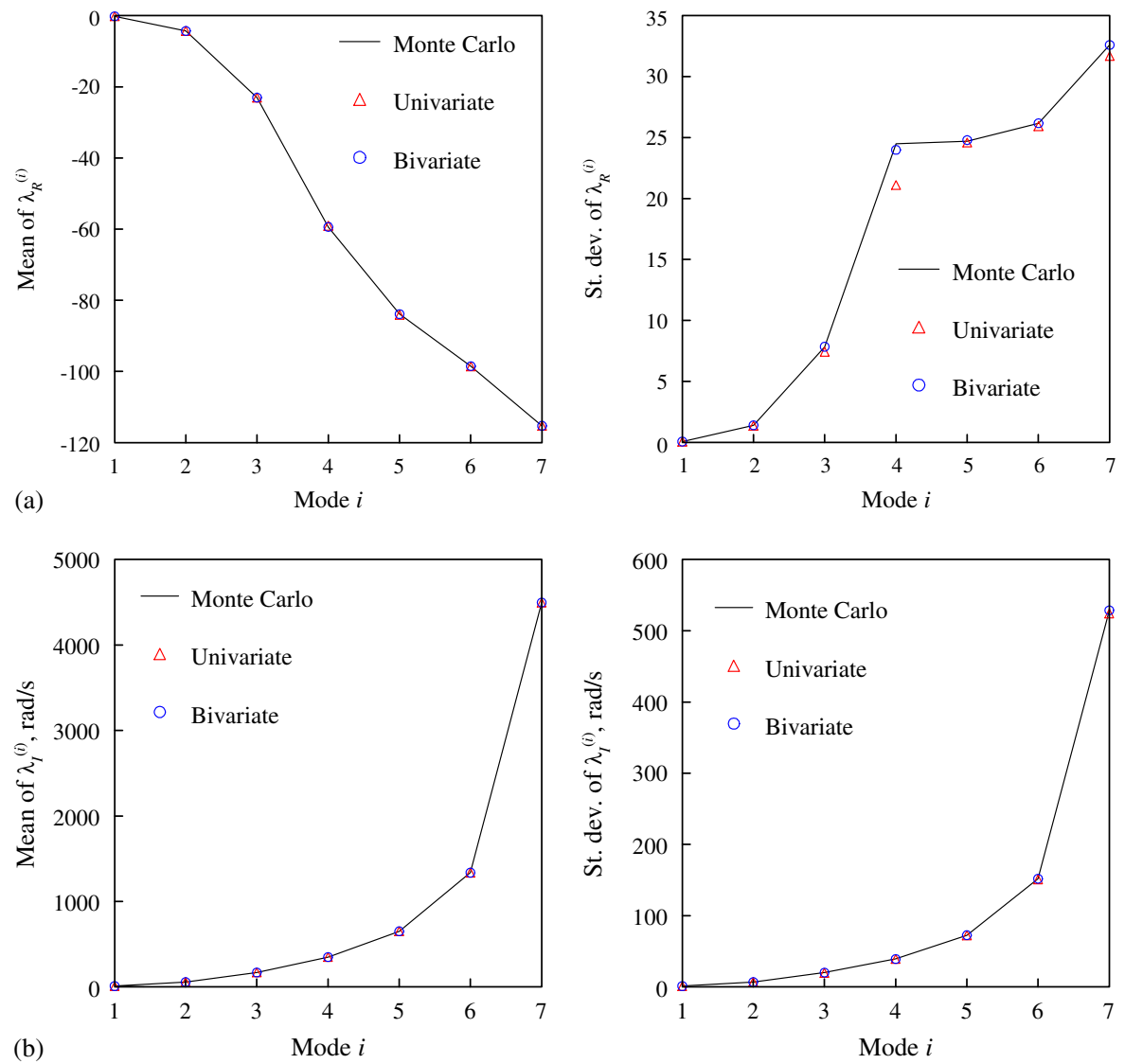

Figure 3. Second-moment properties of eigenvalues of beam: (a) real part; and (b) imaginary part.

standard deviations of real parts $\lambda_{R}^{(i)}(\mathbf{X}) ; i=1, \ldots, 7$ and imaginary parts $\lambda_{I}^{(i)}(\mathbf{X}) ; i=1, \ldots, 7$, respectively, as a function of mode $i$, obtained by the univariate and bivariate decomposition methods and the direct Monte Carlo simulation. The sample size for the direct Monte Carlo simulation and the decomposition method was 100000 . Compared with the direct Monte Carlo simulation, both versions of the decomposition method provide excellent estimates of second-moment properties of eigenvalues. The marginal probability densities of seven real and seven imaginary parts of eigenvalues, respectively, plotted in Figures 4 and 5, also indicate a good agreement between results from the decomposition and direct Monte Carlo methods. As expected, the bivariate method is more accurate than the univariate method.

Corresponding to 14 eigenvalues, there are also 14 complex eigenvectors in conjugate pairs, denoted by $\boldsymbol{\phi}^{(i)}(\mathbf{X})=\boldsymbol{\phi}_{R}^{(i)}(\mathbf{X}) \pm \sqrt{-1} \phi_{I}^{(i)}(\mathbf{X}) ; i=1, \ldots, 7$. Each eigenvector has 14 elements. Consider the real part $\boldsymbol{\phi}_{R}^{(i)}(\mathbf{X}) \equiv\left\{\phi_{R, 1}^{(i)}, \ldots, \phi_{R, 7}^{(i)}, \phi_{R, 8}^{(i)}, \ldots, \phi_{R, 14}^{(i)}\right\}^{\mathrm{T}}$, where the first seven elements define the relative displacements of co-ordinates $y_{1}, \ldots, y_{7}$ and the second seven define the relative velocities of the same co-ordinates $\dot{y}_{1}, \ldots, \dot{y}_{7}$. Figures $6(\mathrm{a})$ and (b) show the mean and standard 

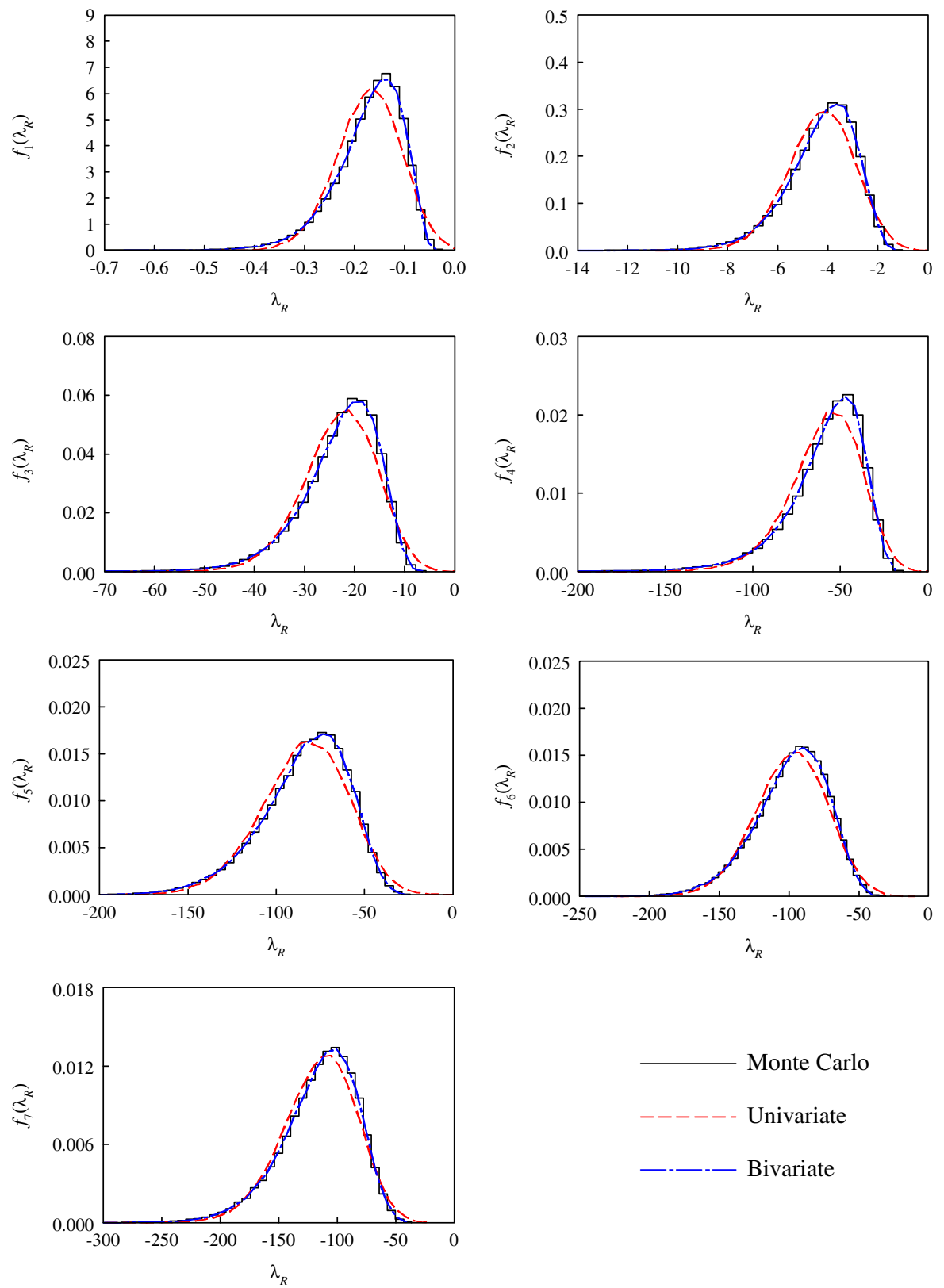

Figure 4. Marginal probability densities of real parts of eigenvalues of beam.

deviation, respectively, of $\left\{\phi_{R, 1}^{(i)}, \ldots, \phi_{R, 7}^{(i)}\right\}^{\mathrm{T}}$ for the first three modes. The agreement between decomposition methods and direct Monte Carlo estimates is again excellent.

The univariate and bivariate decomposition methods require 37 and 613 solutions, respectively, of the matrix characteristic equation, whereas 100000 such solutions (sample size) are involved 

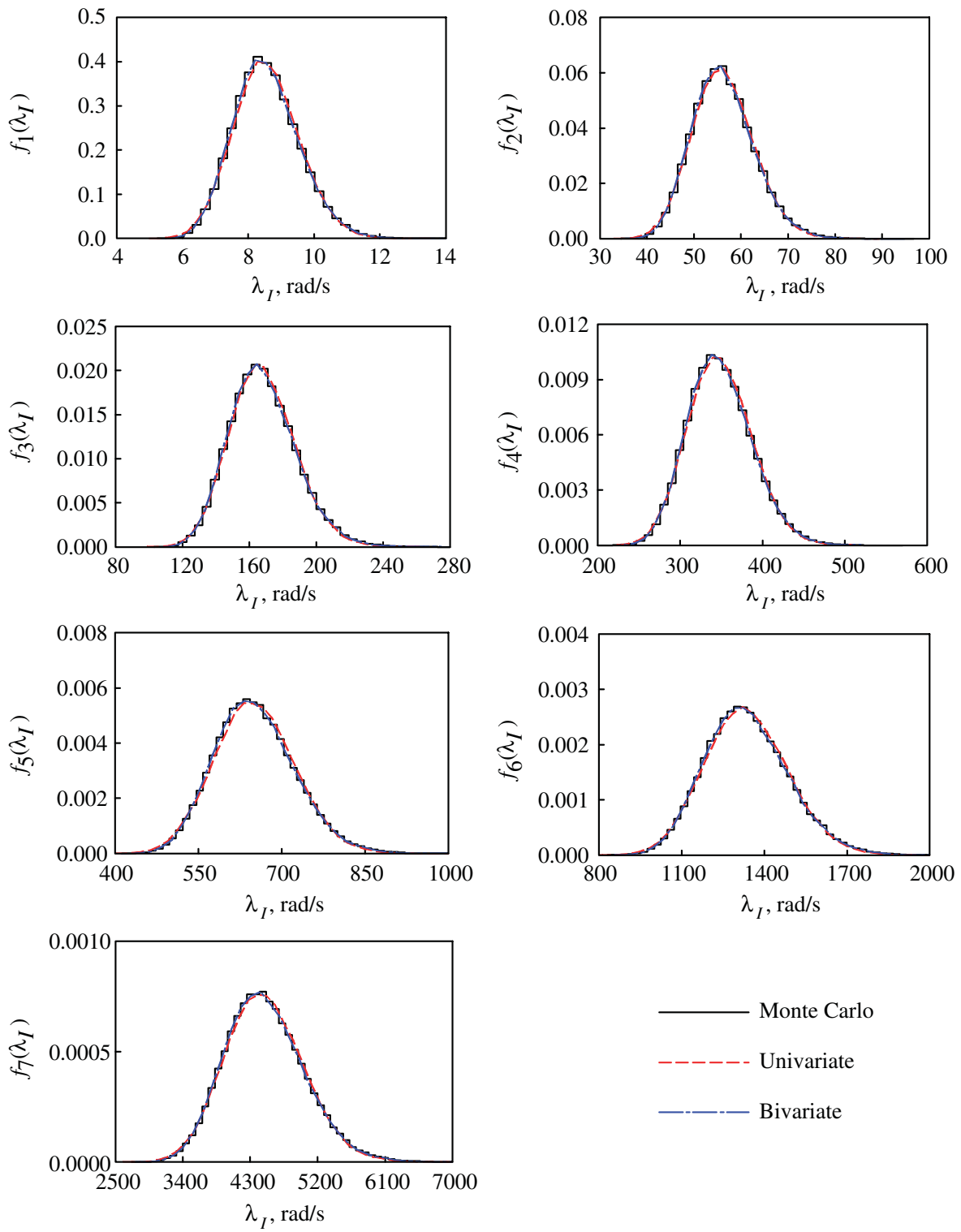

Figure 5. Marginal probability densities of imaginary parts of eigenvalues of beam.

in the direct Monte Carlo simulation. However, these differences, although significant, are less meaningful given that the random matrices are only $7 \times 7$. An example where such difference has a major practical significance is demonstrated next. 

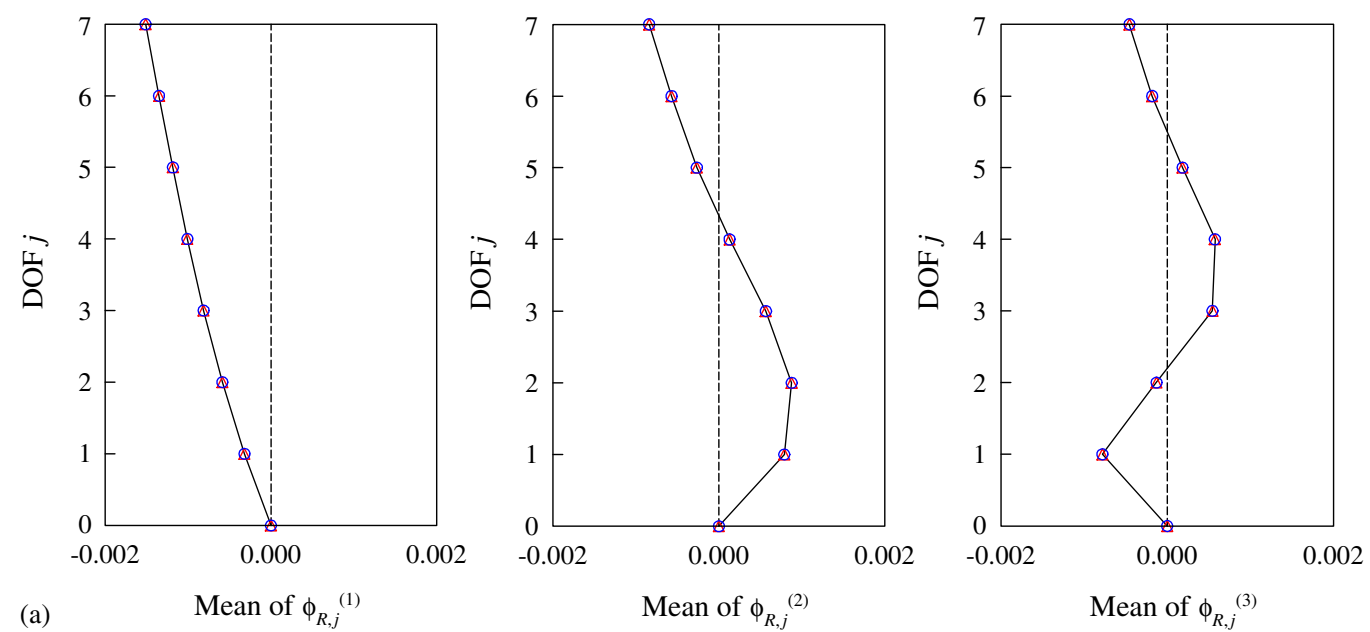

(a)

Mean of $\phi_{R, j}{ }^{(1)}$

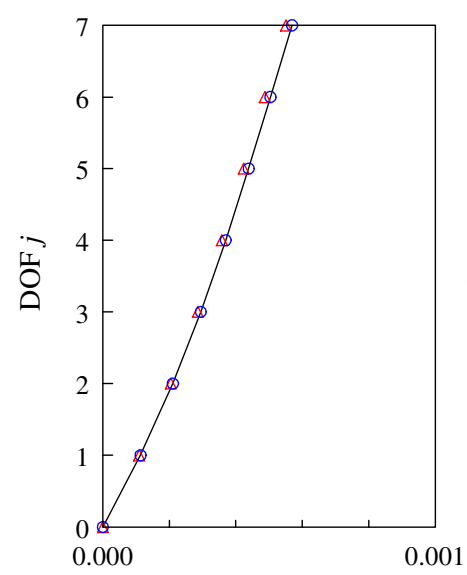

(b)

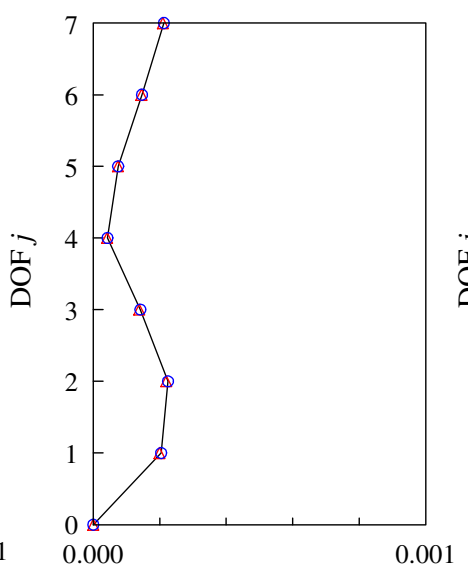

St. dev. of $\phi_{R, j}{ }^{(2)}$

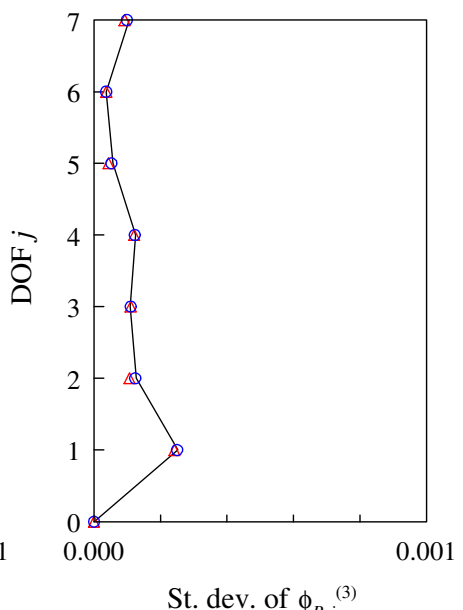

Figure 6. Second-moment properties of real parts of first three eigenvectors of beam: (a) mean; and (b) standard deviation (line $=$ Monte Carlo, open triangle = univariate, open circle $=$ bivariate).

\subsection{Example 3-brake squeal analysis}

The final example of this paper illustrates dynamics of an industrial-scale disc brake system, which defines an eigenvalue problem heavily studied in the automotive industry for understanding brake squeal performance [25]. The probabilistic model developed was applied to evaluate how uncertainties in material properties and frictional coefficient impact brake squeal performance. The brake system consists of a rotor surrounded by two pads on both sides, as shown in Figure 7. The rotor has a diameter of $288 \mathrm{~mm}$, a thickness of $20 \mathrm{~mm}$, and is made of isotropic cast iron with random elastic modulus $E_{\mathrm{c}}=125 X_{1} \mathrm{GPa}$. The pads are made of an anisotropic, organic, frictional material with non-zero random moduli $D_{1111}=D_{2222}=5.94 X_{2} \mathrm{GPa}, D_{1122}=0.76 X_{2} \mathrm{GPa}$, 


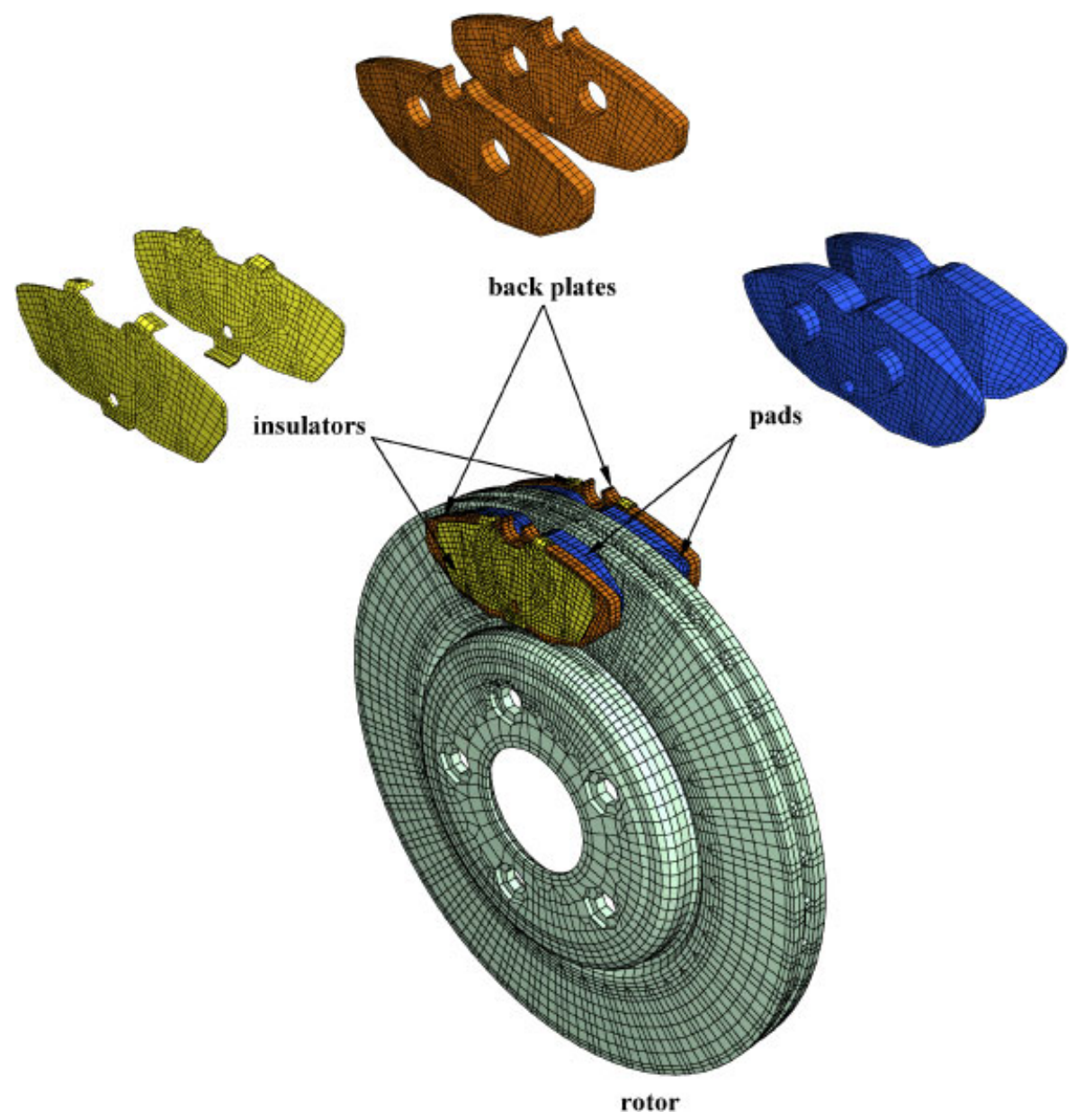

Figure 7. A finite element model of disc brake system with various components.

$D_{1133}=D_{2233}=0.98 X_{2} \mathrm{GPa}, D_{3333}=2.27 X_{2} \mathrm{GPa}, D_{1212}=2.59 X_{2} \mathrm{GPa}$, and $D_{1313}=D_{2323}=$ $1.18 X_{2} \mathrm{GPa}$. Two back plates and insulators, positioned behinds the pads, are made of isotropic steel with random modulus $E_{\mathrm{s}}=207 X_{3} \mathrm{GPa}$. The mass density of the rotor material is $\rho_{\mathrm{c}}=$ $7.2 \times 10^{-6} X_{4} \mathrm{~kg} / \mathrm{mm}^{3}$ and the frictional coefficient between rotor and pad is $f_{\mathrm{r}}=X_{5}$. Table II defines the statistical properties of $\mathbf{X}=\left\{X_{1}, \ldots, X_{5}\right\}^{\mathrm{T}} \in \mathbb{R}^{5}$. The lognormal distribution of $\mathbf{X}$ was defined based on expert judgment. The remaining deterministic parameters are as follows: mass density of steel $=7.82 \times 10^{-6} \mathrm{~kg} / \mathrm{mm}^{3}$; mass density of pad $=2.51 \times 10^{-6} \mathrm{~kg} / \mathrm{mm}^{3}$; and Poisson ratios of the back plate, insulator, and rotor $=0.28,0.29$, and 0.24 , respectively. No damping was included $(\mathbf{C}=\mathbf{0})$ in the present analysis. A finite element model of the disc brake system involving 27,481 C3D6 and C3D8I elements from the ABAQUS commercial code is shown in Figure 7. The total number of degrees of freedom is 881460 . 
Table II. Statistical properties of random input for brake squeal analysis.

\begin{tabular}{lccc}
\hline Random variable* $^{*}$ & Mean & Coefficient of variation & Probability distribution \\
\hline$X_{1}$ & 1 & 0.1 & Lognormal \\
$X_{2}$ & 1 & 0.1 & Lognormal \\
$X_{3}$ & 1 & 0.15 & Lognormal \\
$X_{4}$ & 1 & 0.15 & Lognormal \\
$X_{5}$ & Varies $^{\dagger}$ & 0.2 & Lognormal \\
\hline
\end{tabular}

*All random variables are statistically independent.

$\dagger$ Varies as $0.5,0.3$, and 0.1 .

The disc brake analysis was conducted in three steps. First, a contact between the rotor and the pads was established by applying pressure to the external surface of the insulators. Second, a rotational velocity of $5 \mathrm{rad} / \mathrm{s}$, representative of braking at low speed, was imposed to create a steady-state motion. The resulting equation of motion, described by Equation (2), involves an effective stiffness matrix $\mathbf{K}(\mathbf{X})-\mathbf{K}_{\mathrm{f}}(\mathbf{X})$, where the addition of the friction-induced component $\mathbf{K}_{\mathrm{f}}(\mathbf{X})$ destroys the symmetry, leading to complex modes even when there is no damping. Third, the subspace projection technique embedded in the ABAQUS code was employed to extract complex eigenvalues in the steady-state condition.

In calculating eigenvalues, both deterministic and stochastic analyses were performed. For the deterministic eigenvalue analysis, all previously defined random input except the frictional coefficient were fixed at their mean values. Two separate eigensolutions, one without friction $\left(f_{\mathrm{r}}=0\right)$ and the other with friction $\left(f_{\mathrm{r}}=0.5\right)$, were obtained. When $f_{\mathrm{r}}=0$, the stiffness matrix is symmetric and the system possesses only real modes. In the absence of friction, the 6th and 7 th mode shapes, displayed in Figure 8(a), have frequencies of 1945.6 and $1957.2 \mathrm{~Hz}$, respectively. These two, closely spaced, neighbouring modes merge when the friction is raised to $f_{\mathrm{r}}=0.5$, leading to the mode shapes (real part) in Figure 8(b) with a common frequency of $1953.8 \mathrm{~Hz}$. More importantly, a complex eigenvalue associated with the 7th mode, one of the two merged modes, has a positive real part, which makes the mode unstable. Similar instabilities occur at higher frequencies of $3040.5,8026.0$, and $8997.6 \mathrm{~Hz}$ when $f_{\mathrm{r}}=0.5$. All of these instabilities can be identified by plotting the real part versus the imaginary part (frequency) of the eigenvalues, as presented in Figure 9 for the first 55 eigenvalues evaluated here. These unstable modes are frequently attributed to the dynamic instability of a disc brake system, creating unwanted noise, commonly known as brake squeal.

For the random input $\mathbf{X}$ with its statistical properties listed in Table II and $\mathbb{E}\left[f_{\mathrm{r}}\right]=\mathbb{E}\left[X_{5}\right]=0.5$, the univariate method was employed to evaluate probabilistic characteristics of eigensolutions. The calculation of eigenvalues for a given input is equivalent to performing a large-scale finite element analysis (ABAQUS). Therefore, computational efficiency is a major practical requirement in solving the disc brake random eigenvalue problem. Figures 10(a) and (b) present marginal probability densities of the real and imaginary (frequency) parts, respectively, of the first unstable mode. These probability densities quantify the effect of random input on the variability of important modal properties of a disc brake system. Marginal densities of remaining unstable frequencies can be developed similarly. 

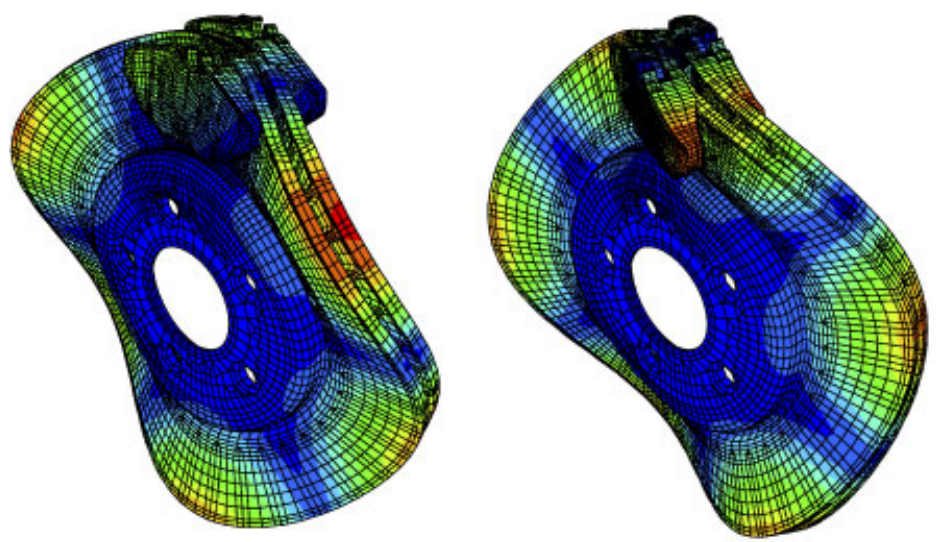

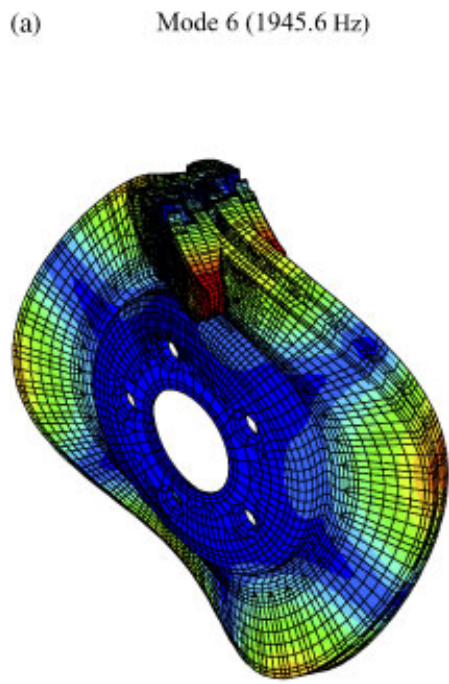

(b)

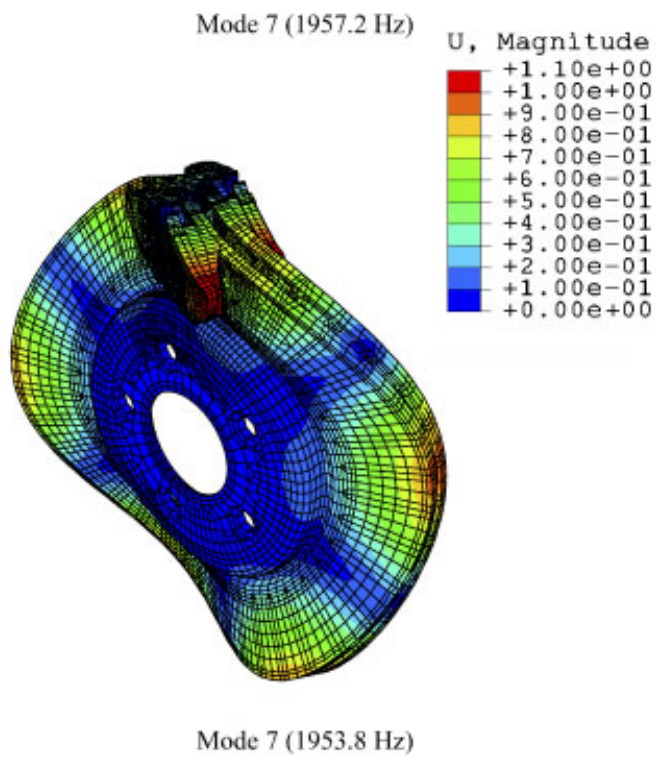

Figure 8. Effect of friction on 6th and 7th modal properties of disc brake system: (a) closely spaced modes $\left(f_{\mathrm{r}}=0\right)$; and (b) merged modes $\left(f_{\mathrm{r}}=0.5\right)$.

For a disc brake system possessing $N_{\mathrm{u}}$ unstable complex modes comprising complex eigenvalues $\lambda_{\mathrm{u}}^{(i)}(\mathbf{X}) ; i=1, \ldots, N_{\mathrm{u}}$, consider a stochastic instability index $U(\mathbf{X})$, defined as $U(\mathbf{X}) \equiv \sum_{i=1}^{N_{\mathrm{u}}}-$ $2 \operatorname{Re}\left[\lambda_{\mathrm{u}}^{(i)}(\mathbf{X})\right] /\left|\operatorname{Im}\left[\lambda_{\mathrm{u}}^{(i)}(\mathbf{X})\right]\right|$. The instability index, which is non-positive, represents a cumulative measure of damping ratios associated with all unstable modes. The magnitude of the instability index signifies brake squeal tendency. Using samples generated from the univariate method, Figure 11 plots distribution functions of $U(\mathbf{X})$ for three different values of $\mathbb{E}\left[f_{\mathrm{r}}\right]=\mathbb{E}\left[X_{5}\right]=0.1,0.3$, and 0.5 . The trend in these distribution functions suggests that the probability of brake squeal can be significantly reduced by lowering the mean frictional coefficient, however, with increasing the brake pressure. A similar stabilization process can be developed by modifying the geometry and material properties of the brake components. 


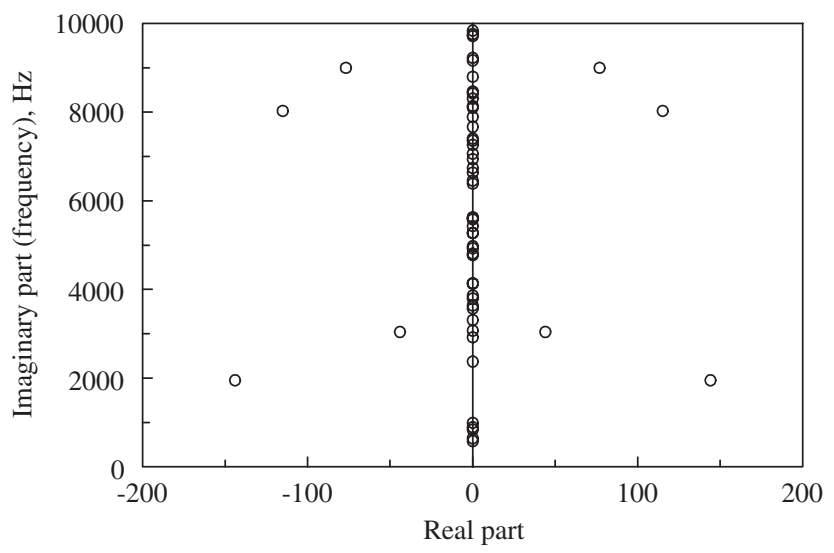

Figure 9. Complex eigenvalues of disc brake system for mean input $\left(f_{\mathrm{r}}=0.5\right)$.
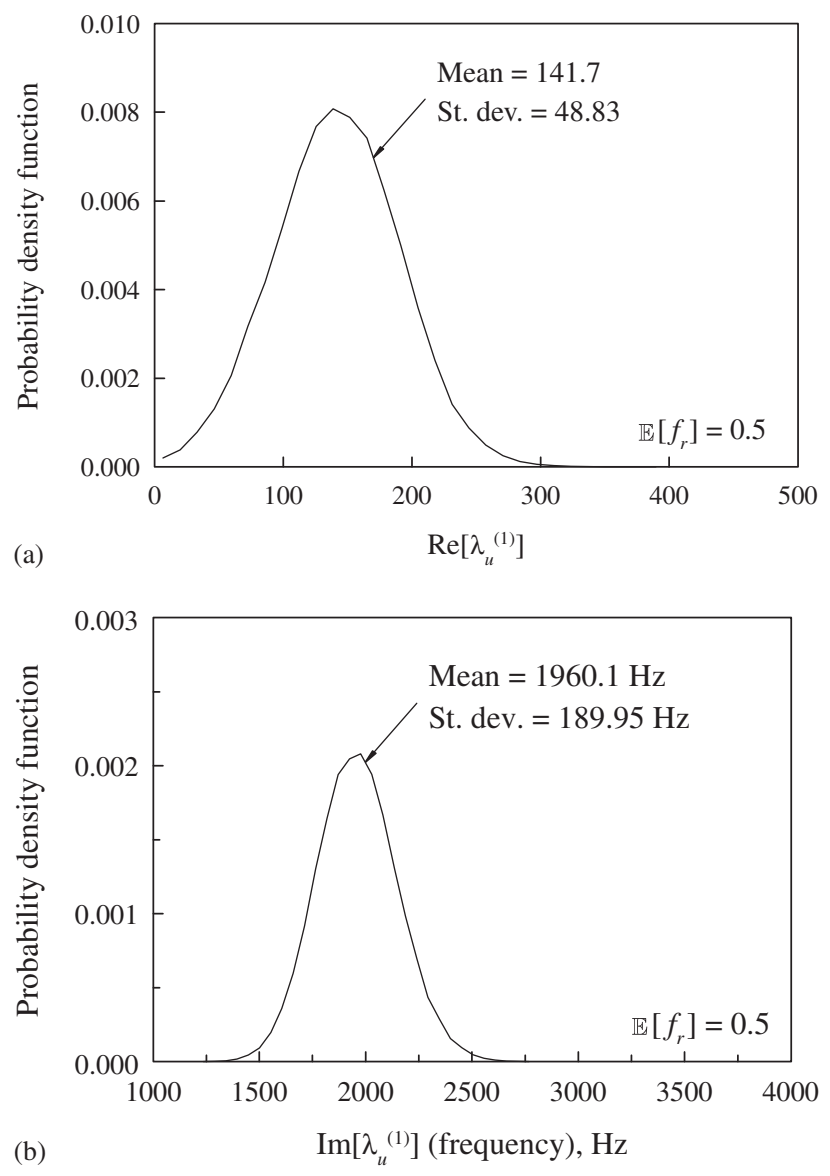

Figure 10. Probability density of complex eigenvalue of disc brake system at first unstable mode by the univariate method: (a) real part; and (b) imaginary part (frequency). 


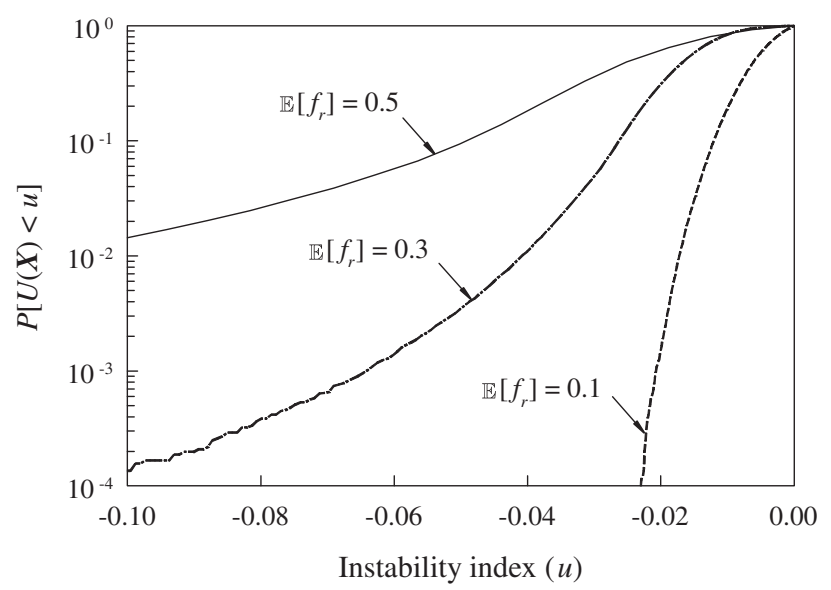

Figure 11. Cumulative distribution function of instability index by the univariate method.

For computational effort involving $N=n=5$, only 21 finite element analyses were required by the univariate method. Since the decomposition method yields an explicit eigenvalue approximation, an arbitrarily large sample size, 100000 in this example, was selected to perform the embedded Monte Carlo analysis. Due to the computational expense inherent to ABAQUS analysis, no direct Monte Carlo analysis was feasible for this problem.

\section{CONCLUSION}

A dimensional decomposition method was developed for calculating the probabilistic characteristics of complex-valued eigenvalues and eigenvectors of linear, stochastic, dynamic systems. The method is based on: (1) a finite, hierarchical decomposition allowing lower-dimensional approximations of eigensolutions; (2) Lagrange interpolation of lower-dimensional component functions; and (3) Monte Carlo simulation. The effort in finding the probabilistic characteristics of eigensolutions can be viewed as performing eigenvalue analyses at selected deterministic input defined by sample points. Compared with the commonly used perturbation method, neither the assumption of small input variability nor the calculation of the derivatives of eigensolutions is required by the method developed. Hence, the method can be easily adapted for solving random eigenvalue problems involving third-party, commercial, finite-element codes. The proposed method was applied to a nonproportionally damped spring-mass-damper system, a non-proportionally damped cantilever beam, and an industrial-scale disc brake system with an unsymmetric stiffness matrix. Results indicate that the decomposition method provides excellent estimates of the moments and/or probability densities of eigenvalues and eigenvectors for various cases including large statistical variations of input. The computational efforts required by the univariate and bivariate decomposition method are linear and quadratic, respectively, with regard to the number of random variables involved. Therefore, the method developed is also computationally efficient when compared with the direct Monte Carlo simulation. 


\section{APPENDIX A}

For the discrete model of the beam, the $7 \times 7$ mass, damping, and stiffness matrices are

$$
\mathbf{M}(\mathbf{X})=\frac{M(\mathbf{X}) l}{4}\left[\begin{array}{lllllll}
0 & 0 & 0 & 0 & 0 & 1 & 1 \\
0 & 0 & 0 & 0 & 1 & 4 & 3 \\
0 & 0 & 0 & 1 & 4 & 8 & 5 \\
0 & 0 & 1 & 4 & 8 & 12 & 7 \\
0 & 1 & 4 & 8 & 12 & 16 & 9 \\
1 & 4 & 8 & 12 & 16 & 20 & 11 \\
4 & 8 & 12 & 16 & 20 & 24 & 13
\end{array}\right]
$$

and

$$
\begin{aligned}
\mathbf{K}(\mathbf{X})= & \frac{M(\mathbf{X}) g}{2} \\
& \times\left[\begin{array}{ccccccc}
0 & 0 & 0 & 0 & \bar{k}_{6} & -2 \bar{k}_{6}+1 & \bar{k}_{6}-1 \\
0 & 0 & 0 & \bar{k}_{5} & -2 \bar{k}_{5}+3 & \bar{k}_{5}-2 & -1 \\
0 & 0 & \bar{k}_{4} & -2 \bar{k}_{4}+5 & \bar{k}_{4}-2 & -2 & -1 \\
0 & \bar{k}_{3} & -2 \bar{k}_{3}+7 & \bar{k}_{3}-2 & -2 & -2 & -1 \\
\bar{k}_{2} & -2 \bar{k}_{2}+9 & \bar{k}_{2}-2 & -2 & -2 & -2 & -1 \\
-2 \bar{k}_{1}+11 & \bar{k}_{1}-2 & -2 & -2 & -2 & -2 & -1 \\
\bar{K}-2 & -2 & -2 & -2 & -2 & -2 & -1
\end{array}\right]
\end{aligned}
$$

respectively, where $\bar{k}_{i}(\mathbf{X})=2 k_{i}(\mathbf{X}) /(M(\mathbf{X}) g l), \bar{K}(\mathbf{X})=2 K(\mathbf{X}) /(M(\mathbf{X}) g l)$, and $g$ is the acceleration due to the gravity. 


\section{ACKNOWLEDGEMENTS}

The author would like to acknowledge financial support from the U.S. National Science Foundation under Grant No. DMI-0355487. The author also thanks Dr A. Bajer of ABAQUS, Inc. for providing valuable insights on disc brake analysis in Example 3.

\section{REFERENCES}

1. Boyce WE. Probabilistic Methods in Applied Mathematics I. Academic Press: New York, NY, 1968.

2. Shinozuka M, Astill CJ. Random eigenvalue problems in structural analysis. AIAA Journal 1972; 10(4):456-462.

3. Zhang J, Ellingwood B. Effects of uncertain material properties on structural stability. Journal of Structural Engineering 1995; 121(4):705-714.

4. Mehlhose S, Vom Scheidt J, Wunderlich R. Random eigenvalue problems for bending vibrations of beams. Zeitschrift für Angewandte Mathematik und Mechanik 1999; 79:693-702.

5. Grigoriu M. A solution of the random eigenvalue problem by crossing theory. Journal of Sound and Vibration 1992; 158(1):69-80.

6. Nair PB, Keane AJ. An approximate solution scheme for the algebraic random eigenvalue problem. Journal of Sound and Vibration 2003; 260(1):45-65.

7. Adhikari S, Friswell MI. Random matrix eigenvalue problems in structural dynamics. International Journal for Numerical Methods in Engineering 2006, in press.

8. Ghosh D, Ghanem RG, Red-Horse J. Analysis of eigenvalues and modal interaction of stochastic systems. AIAA Journal 2005; 43(10):2196-2201.

9. Rahman S. A solution of the random eigenvalue problem by a dimensional decomposition method. International Journal for Numerical Methods in Engineering 2006; 67:1318-1340.

10. Caughey TK, O'Kelly MEJ. Classical normal modes in damped linear dynamic systems. ASME Journal of Applied Mechanics 1965; 32:583-588.

11. Adhikari S. Complex modes in stochastic systems. Advances in Vibration Engineering 2004; 3(1):1-11.

12. $\mathrm{Xu} \mathrm{H}$, Rahman S. A generalized dimension-reduction method for multi-dimensional integration in stochastic mechanics. International Journal for Numerical Methods in Engineering 2004; 61:1992-2019.

13. Xu H, Rahman S. Decomposition methods for structural reliability analysis. Probabilistic Engineering Mechanics 2005; 20:239-250.

14. Hoeffding W. A class of statistics with asymptotically normal distributions. Annals of Mathematical Statistics 1948; 19:293-325.

15. Efron B, Stein C. The Jackknife estimate of variance. Annals of Statistics 1981; 9:586-596.

16. Sobol IM. Theorems and examples on high dimensional model representations. Reliability Engineering and System Safety 2003; 79:187-193.

17. Rabitz H, Alis O. General foundations of high dimensional model representations. Journal of Mathematical Chemistry 1999; 25:197-233.

18. Li G, Wang SW, Rabitz H. Practical approaches to construct RS-HDMR component functions. Journal of Physical Chemistry A 2002; 106:8721-8733.

19. Li G, Rosenthal C, Rabitz H. High dimensional model representations. Journal of Physical Chemistry A 2001; 105:7765-7777.

20. Li G, Wang SW, Rosenthal C, Rabitz H. High dimensional model representations generated from low dimensional data samples-I. mp-Cut-HDMR. Journal of Mathematical Chemistry 2001; 30:1-30.

21. Puig B, Akian JL. Non-Gaussian simulation using hermite polynomial expansion and maximum entropy principle. Probabilistic Engineering Mechanics 2004; 19:293-305.

22. Newland DE. Mechanical Vibration Analysis and Computation. Wiley: New York, NY, 1989.

23. IMSL Numerical Libraries. User's Guide and Theoretical Manual. Visual Numerics Corporate Headquarters, San Ramon, CA, 2005.

24. ABAQUS. User's Guide and Theoretical Manual, Version 6.5. Hibbitt, Karlsson, and Sorenson, Inc.: Pawtucket, RI, 2005.

25. Bajer A, Belsky V, Zeng LJ. Combining a nonlinear static analysis and complex eigenvalue extraction in brake squeal simulation. Proceedings of 21st Annual Brake Colloquium and Exhibition, SAE Paper no. 2003-01-3349. Hollywood, FL, 2003. 